\title{
Contrast and Classify: Training Robust VQA Models
}

\author{
Yash Kant $^{1 *}$ Abhinav Moudgil $^{1} \quad$ Dhruv Batra $^{1,2}$ Devi Parikh ${ }^{1,2} \quad$ Harsh Agrawal $^{1}$ \\ ${ }^{1}$ Georgia Institute of Technology $\quad{ }^{2}$ Facebook AI Research
}

\begin{abstract}
Recent Visual Question Answering (VQA) models have shown impressive performance on the VQA benchmark but remain sensitive to small linguistic variations in input questions. Existing approaches address this by augmenting the dataset with question paraphrases from visual question generation models or adversarial perturbations. These approaches use the combined data to learn an answer classifier by minimizing the standard cross-entropy loss. To more effectively leverage augmented data, we build on the recent success in contrastive learning. We propose a novel training paradigm (ConClaT) that optimizes both cross-entropy and contrastive losses. The contrastive loss encourages representations to be robust to linguistic variations in questions while the cross-entropy loss preserves the discriminative power of representations for answer prediction.

We find that optimizing both losses - either alternately or jointly - is key to effective training. On the VQARephrasings [45] benchmark, which measures the VQA model's answer consistency across human paraphrases of a question, ConClaT improves Consensus Score by 1.63\% over an improved baseline. In addition, on the standard VQA 2.0 benchmark, we improve the VQA accuracy by $0.78 \%$ overall. We also show that ConClaT is agnostic to the type of data-augmentation strategy used.
\end{abstract}

\section{Introduction}

Visual Question Answering (VQA) refers to the task of automatically answering free-form natural language questions about an image. For VQA systems to work reliably when deployed in the wild, for applications such as assisting visually impaired users, they need to be robust to different ways a user might ask the same question. For example, VQA models should produce the same answer for two paraphrased questions - "What is in the basket?" and "What is contained in the basket?" since their semantic meaning is the same. While significant progress has been made towards building more accurate VQA systems, these models remain

${ }^{*}$ Correspondence to ysh. kantegmail.com

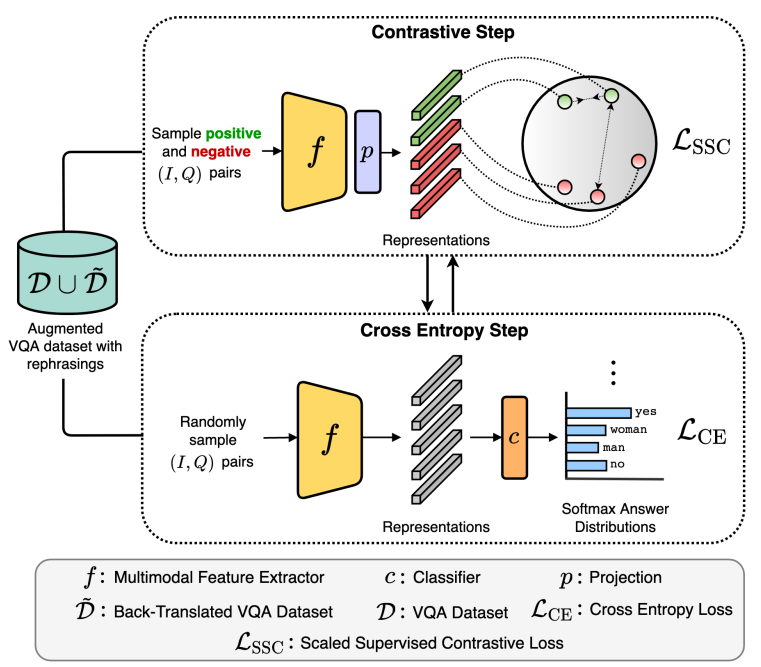

Figure 1: We make VQA model robust to question paraphrases using a training paradigm ConClaT that minimizes contrastive and cross-entropy losses together. Contrastive learning step pulls representations of positive samples corresponding to paraphrased questions closer together while pushing those with different answers farther apart. Crossentropy step makes these representations discriminative to help model answer visual questions accurately.

brittle to minor linguistic variations in the input question.

To make VQA systems robust, existing approaches [45, 48] have trained VQA systems [24] by augmenting the training data with different variations of the input question. For instance, VQA-CC [45] use a visual question generation (VQG) model to generate paraphrased question given an image and answer. Generally, these models fuse image and question features into a joint vision and language $(\mathrm{V}+\mathrm{L})$ representation followed by a standard softmax classifier to produce answer probabilities and are optimized by minimizing the cross-entropy loss. Unfortunately, crossentropy loss treats every image-question pair independently and fails to exploit the information that some questions in the augmented dataset are paraphrases of each other.

We overcome this limitation by using a contrastive loss InfoNCE [36] that encourages joint $\mathrm{V}+\mathrm{L}$ (Vision and Language) representations obtained from samples whose ques- 
tions are paraphrases of each other to be closer while pulling apart the $\mathrm{V}+\mathrm{L}$ representations of samples with different answers. As we operate in a supervised setting, we choose Supervised Contrastive Loss (SCL) [26] which extends InfoNCE by utilizing the label information to bring samples from the same class (ground-truth answer) together. We introduce a variant of the SCL which emphasizes rephrased image-question pairs over pairs that are entirely different but have the same answer. Our proposed training paradigm, ConClaT (Contrast and Classify Training), minimizes SCL and cross-entropy loss together to learn better vision and language representations as shown in Fig.1. Minimizing the contrastive loss encourages representations to be robust to linguistic variations in questions while the cross-entropy loss preserves the discriminative power of the representations for answer classification. Instead of pretraining with SCL, then fine-tuning with cross-entropy loss as in [26], we find that minimizing the two losses either alternately or jointly by constructing loss-specific mini-batches helps learn better representations. For contrastive loss, we carefully curate mini-batches by sampling various types of negatives and positives given a reference sample.

We show the efficacy of our training paradigm across two rephrasing (i.e., data-augmentation) strategies. Using rephrasings obtained from a VQG model proposed in [45], our approach outperforms a baseline that simply treats these rephrasings as additional samples and ignores the link between question and its paraphrases. We noticed that the VQG model fails to produce a diverse set of rephrasings for a question. Hence, we use Back-translation to obtain question rephrasings. Back-translation [15] involves translating an input sentence from one language to another and then translating it back into the original language using a pair of machine translation models (e.g. en-fr and $f r-e n)$. We find that Back-translation preserves the semantic meaning of the question while generating syntactically diverse question. Utilizing the publicly available collection of neural machine translation models in HuggingFace [53], we generate numerous rephrasings of every question. Then, we filter poor/irrelevant rephrasings with a sentence similarity model [42] and store 3 rephrasings per original question of VQA v2.0 dataset without any manual supervision.

We extensively ablate ConClaT with alternate [8], joint and pretrain-finetune [26] training schemes, and compare with previously proposed triplet [39] and margin-based losses [60] . We evaluate on the VQA Rephrasings benchmark [45] which measures the model's answer consistency across several rephrasings of a question. ConClaT improves Consensus Score by $1.63 \%$ over an improved baseline. In addition, on the standard VQA 2.0 benchmark, we improve VQA accuracy by $0.78 \%$ overall. It is also worth noting that VQA models trained using ConClaT perform better than existing approaches across both the aforementioned data- augmentation strategies - Back-translation and VQG.

\section{Related Work}

Models for VQA. Several models have been proposed for Visual Question Answering which fuse CNN grid features and LSTM features with different forms of attention [34, 56, 16, 23]. Bottom-Up and Top-Down [6] proposed to learn attention over object regions obtained from a pretrained object detector and subsequent works [27, 58, 24] introduced various ways to fuse image and language representations. Recent works [32, 33, 46, 29, 46, 47, 13] use multi-modal transformers to learn visuo-linguistic representations from object detector features and BERT question features [14]. We use the multi-modal transformer architecture similar to UNITER [13] for all our experiments.

Robustness of VQA Models. Robustness of VQA models with respect to multi-modal vision and language input has been studied in great detail. [18, 59] proposed balanced datasets to ensure models don't overfit to language while answering visual questions. C-VQA [4] and VQA$\mathrm{CP}$ [3] datasets were proposed to test robustness against changing question-answer distributions. SQUINT [44] encouraged consistency between reasoning questions and associated sub-questions. Our work focuses on robustness to question paraphrases in VQA-Rephrasings [45] that were collected from human annotators. VQA-CC [45] trained a Visual Question Generation (VQG) model to generate paraphrases of questions to augment the training dataset while VQA-Aug [48] augmented the training dataset by generating paraphrases of questions via back-translation. We show that these data augmentation techniques can be better utilized via ConClaT to build robust and accurate VQA models. Concurrent to our work, Whitehead et al. [51] propose a rule-based mechanism to generate question paraphrases for VQA. They constrain their model architecture to be modular [7] and use module-level loss to improve consistency. In contrast, our approach is agnostic to model architecture.

Various works [3, 2, 60, 39] made VQA models robust to language bias (For example, "What is the color of $x$ " will always produce 'blue' irrespective of $x$ ). Recent works [49, 1, 9, 37] also studied robustness from counterfactual answering lens - answer should change according to the change in semantic content of the question or image. Our work, on the other hand, focuses on robustness to syntactic variations in questions.

Paraphrase Generation in NLP. There has been significant work in the area of Natural Language Processing (NLP) for generating paraphrases of a sentence using LSTM networks [40], Deep Reinforcement Learning [31], Variational Autoencoders [19] and Transformers [50]. However, these works require supervision in the form of paraphrase pairs. In order to mitigate this limitation of labelled data, Neural Machine Translation (NMT) 
models have been used to generate paraphrases in a selfsupervised fashion via back-translation [35, 52]. We build on top of these works and use state-of-the-art NMT models from HuggingFace [53] to generate paraphrases for visual questions without any supervision.

Contrastive Learning. There has been recent interest in the use of Contrastive Learning for learning visual representations in a self-supervised manner [54, 22, 21, 10, 12, 11, 41]. Going beyond Image Classification, recently, [20] used contrastive learning for phrase grounding. They used the InfoNCE loss [36] to learn a compatibility function between a set of region features from an image and contextualized word representations. In contrast, we want to learn representations which are robust to linguistic variations in the question for VQA.

To utilize label information in contrastive losses, [26] proposed Supervised Contrastive Learning (SCL) loss for learning visual representations. We introduce a variant of the SCL which scales the contributions from augmented positive samples (rephrasings in our case) over intra-class positive samples (that have the same answer) using a scaling factor. Morever, our training paradigm optimizes both (cross-entropy and SCL) losses together, whereas [26] follow the pretrain-finetune training scheme. Furthermore, [26] randomly sample positive and negative pairs based on label information, whereas we carefully curate batches by sampling hard-negatives from the dataset. We show how these differences affect performance through a series of ablations in our experiments section.

\section{Preliminaries}

In this section, we introduce the VQA task and the standard cross entropy training of VQA models. We then recap contrastive methods for learning representations [10] and the recently proposed Supervised Contrastive Learning (SCL) [26] setup. We describe our approach in section 4.

VQA. The task of Visual Question Answering (VQA) [5, 18] involves predicting an answer $a$ for a question $q$ about an image $v$. An instance of this problem in the VQA Dataset $\mathcal{D}$ is represented via a tuple $x=(v, q, a), \forall x \in \mathcal{D}$. Recent VQA models [24, 6, 13] take image and question as input and output a joint vision and language $(\mathrm{V}+\mathrm{L})$ representation $\boldsymbol{h} \in \mathcal{R}^{d_{h}}$ using a multi-modal network $f$ :

$$
\boldsymbol{h}=f(v, q)
$$

The $\mathrm{V}+\mathrm{L}$ representation $\boldsymbol{h}$ is then used to predict a probability distribution over the answer space $\mathcal{A}$ with a softmax classifier $f^{c}(\boldsymbol{h})$ learned by minimizing the cross-entropy:

$$
\mathcal{L}_{\mathrm{CE}}=-\log \frac{\exp \left(f^{c}(\boldsymbol{h})[a]\right)}{\sum_{a^{\prime} \in \mathcal{A}} \exp \left(f^{c}(\boldsymbol{h})\left[a^{\prime}\right]\right)}
$$

where $f^{c}(\boldsymbol{h})[a]$ is the logit corresponding to the answer $a$.
Contrastive Learning. Recent works in vision [10] have used contrastive losses to bring representations of two augmented views of the same image (called positives) closer together while pulling apart the representations of two different images (called negatives). The representation $\boldsymbol{h}$ obtained from an image encoder is projected into a $d_{z}$ dimensional hyper-sphere using a projection network $g$ such that $\boldsymbol{z}=g(\boldsymbol{h}) \in \mathcal{R}^{d_{z}}$. Given a mini-batch of size $K$, the image representation $\boldsymbol{h}$ is learned by minimizing the InfoNCE [36] loss which operates on a pair of positives $\left(\boldsymbol{z}_{i}, \boldsymbol{z}_{p}\right)$ and $K-1$ negative pairs $\left(\boldsymbol{z}_{i}, \boldsymbol{z}_{k}\right)$ such that $i, p, k \in[1, K], k \neq i$ as follows:

$$
\mathcal{L}_{\mathrm{NCE}}^{i}=-\log \frac{\exp \left(\Phi\left(\boldsymbol{z}_{i}, \boldsymbol{z}_{p}\right) / \tau\right)}{\sum_{k=1}^{K} \mathbb{1}_{k \neq i} \exp \left(\Phi\left(\boldsymbol{z}_{i}, \boldsymbol{z}_{k}\right) / \tau\right)},
$$

where $\Phi(\boldsymbol{u}, \boldsymbol{v})=\boldsymbol{u}^{\top} \boldsymbol{v} /\|\boldsymbol{u}\|\|\boldsymbol{v}\|$ computes similarity between $\boldsymbol{u}$ and $\boldsymbol{v}$ and $\tau>0$ is a scalar temperature parameter.

A generalization of InfoNCE loss to handle more than one positive-pair was proposed by [26] called Supervised Contrastive Loss (SCL). Given a reference sample $x$, SCL uses class-label information to form a set of positives $\mathcal{X}^{+}(x)$ that contains samples with the same label as $x$. $\mathcal{X}^{+}(x)$ also contains augmented views of the sample because they share the same label as $x$. For a minibatch with $K$ samples, SCL is defined as:

$$
\begin{gathered}
\mathcal{L}_{\mathrm{SC}}^{i}=-\sum_{p=1}^{\left|\mathcal{X}^{+}\left(x_{i}\right)\right|} \log \frac{\exp \left(\Phi\left(\boldsymbol{z}_{i} \cdot \boldsymbol{z}_{p}\right) / \tau\right)}{\sum_{k=1}^{K} \mathbb{1}_{k \neq i} \cdot \exp \left(\Phi\left(\boldsymbol{z}_{i} \cdot \boldsymbol{z}_{p}\right) / \tau\right)} \\
\mathcal{L}_{\mathrm{SC}}=\sum_{i=1}^{K} \frac{\mathcal{L}_{\mathrm{SC}}^{i}}{\left|\mathcal{X}^{+}\left(x_{i}\right)\right|}
\end{gathered}
$$

Overall, $\mathcal{L}_{\mathrm{SC}}^{i}$ tries to bring the representation of samples in $\mathcal{X}^{+}\left(x_{i}\right)$ closer together compared to representations of samples with a different ground-truth label.

\section{Approach}

We now describe our approach, ConClaT, which uses contrastive and cross-entropy training to learn VQA models robust to question paraphrases.

\subsection{Augmented Dataset with Back-translation}

We augment the train set with question paraphrases using 88 different MarianNMT [25] Back-translation model pairs released by HuggingFace [53]. We produce 27 unique rephrasings per question with cosine similarity of 0.88 on average, the similarity is calculated by first encoding the questions via Sentence-BERT [42]. We only select paraphrases that have $\geq 0.95$ similarity with the original question and choose three unique paraphrases randomly from this subset. We use three paraphrases to keep the compute 


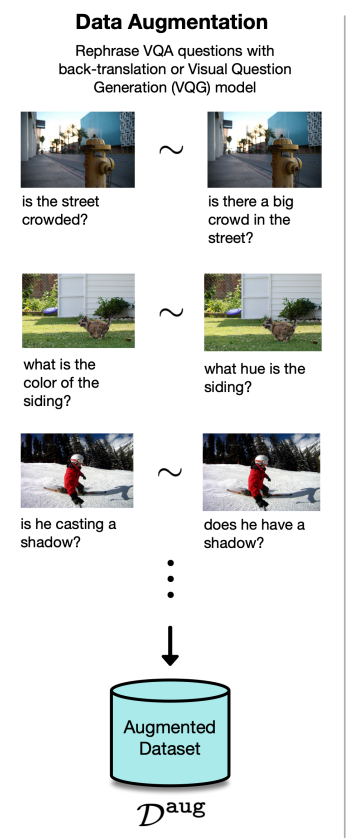

(a)

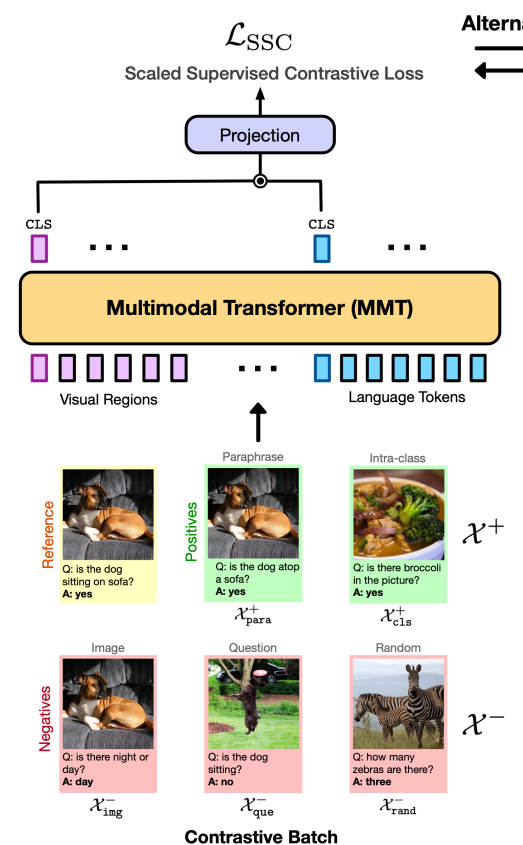

(b)

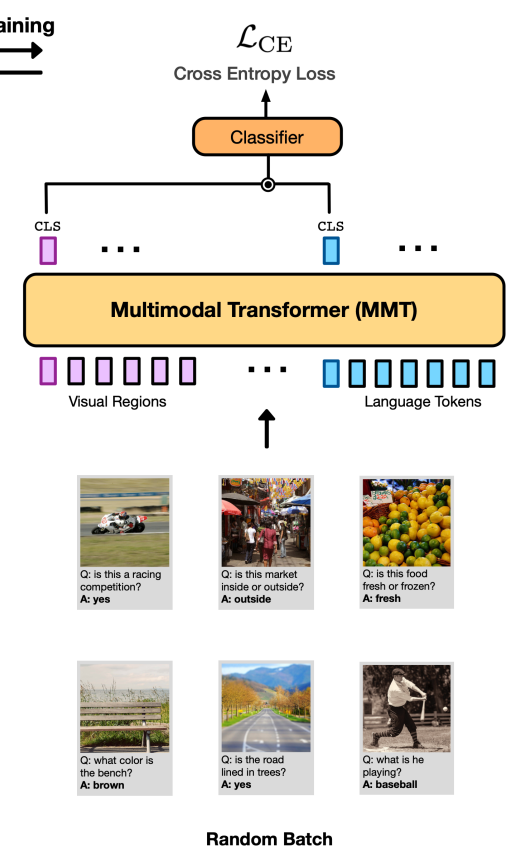

(c)

Figure 2: Overview of ConClaT. (a) We augment the VQA dataset by paraphrasing every question via Back-translation or Visual Question Generation. (b) We carefully curate a contrastive batch by sampling different types of positives and negatives to learn joint $\mathrm{V}+\mathrm{L}$ representations by minimizing scaled supervised contrastive loss $\mathcal{L}_{\text {SSC }}$. (c) Cross Entropy loss $\mathcal{L}_{\mathrm{CE}}$ is optimized with $\mathcal{L}_{\mathrm{SSC}}$.

manageable. Overall, our augmented train set consists of $\sim$ $1.6 \mathrm{M}$ samples.

For a sample $x=(v, q, a) \in \mathcal{D}$, let's denote a set of paraphrases for question $q$ by $\mathcal{Q}(q)$ and the corresponding set of VQA triplets as:

$$
\mathcal{X}_{\text {para }}^{+}(x)=\left\{\left(v, q^{\prime}, a\right) \mid q^{\prime} \in \mathcal{Q}(q)\right\}
$$

As shown in Figure 2(a), we augment the VQA dataset $\mathcal{D}$ with multiple paraphrased samples of a given question and denote the augmented dataset $\mathcal{D}^{\text {aug }}$ as:

$$
\mathcal{D}^{\text {aug }}=\mathcal{D} \bigcup_{x \in \mathcal{D}} \mathcal{X}_{\text {para }}^{+}(x)
$$

\subsection{Scaled Contrastive Loss for VQA}

We would like our VQA model to produce the same and correct answer for a question and its paraphrase given an input image. This motivates us to map joint vision and language $(\mathrm{V}+\mathrm{L})$ representations of an original and paraphrased sample closer to each other. Moreover, since we operate in a supervised setting, following SCL [26] we also pull the joint representations for the questions with the same answer (intra-class positives) closer together while pulling apart the representations of questions with different answers. We define the set of all samples with the same ground truth answer as $x$ by:

$$
\mathcal{X}^{+}(x)=\left\{(\hat{v}, \hat{q}, \hat{a}) \in \mathcal{D}^{\text {aug }} \mid \hat{a}=a\right\}
$$

Note that $\mathcal{X}_{\text {para }}^{+}(x) \subset \mathcal{X}^{+}(x)$ as all question paraphrases have the same answer for a given image but not all questions with the same answer are paraphrases. We refer to samples in set $\mathcal{X}_{\mathrm{cls}}^{+}(x)=\mathcal{X}^{+}(x)-\mathcal{X}_{\text {para }}^{+}(x)$ as intraclass positives and set $\mathcal{X}_{\text {para }}^{+}(x)$ as paraphrased positives w.r.t. $x$ as depicted in Figure 2(b).

Following Eq. (3), all the samples in $\mathcal{X}^{+}\left(x_{i}\right)$ in $\mathcal{L}_{\mathrm{SC}}$ are treated the same. That is, representations from both the paraphrased positives and intra-class positives are brought closer together. To emphasize on the link between question and its paraphrase, we propose a variant of the SCL in Eq. (7) which assigns higher weight to paraphrased positives $\mathcal{X}_{\text {para }}^{+}(x)$ over intra-class positives $\mathcal{X}_{\mathrm{cls}}^{+}(x)$. We introduce a scaling factor $\alpha_{i p}$ in the SCL (Eq. (3)) for a sample $x_{i}$ as follows:

$\mathcal{L}_{\text {SSC }}^{i}=-\sum_{p=1}^{\left|\mathcal{X}^{+}\left(x_{i}\right)\right|} \alpha_{i p} \cdot \log \frac{\exp \left(\Phi\left(\boldsymbol{z}_{i} \cdot \boldsymbol{z}_{p}\right) / \tau\right)}{\sum_{k=1}^{K} \mathbb{1}_{k \neq i} \cdot \exp \left(\Phi\left(\boldsymbol{z}_{i} \cdot \boldsymbol{z}_{p}\right) / \tau\right)}$

$$
\mathcal{L}_{\mathrm{SSC}}=\sum_{i=1}^{K} \frac{\mathcal{L}_{\mathrm{SSC}}^{i}}{\sum_{p} \alpha_{i p}}
$$




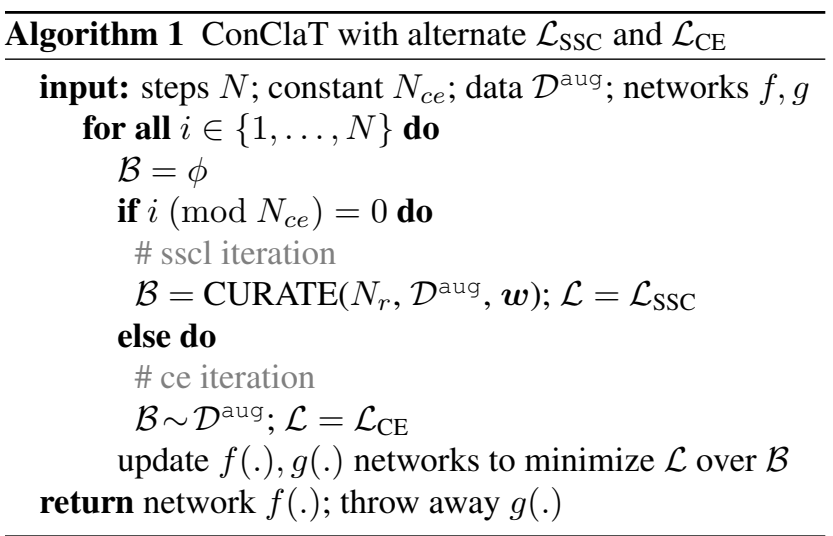

The scaling factor $\alpha_{i p}$ assigns a higher weight $s>1$ to positive samples corresponding to question paraphrases compared to other intra-class positives. Intuitively, because of the higher weight, the loss will penalize the model strongly if it fails to bring the representations of a question and its paraphrase closer. We define $\alpha_{i p}$ as:

$$
\alpha_{i p}= \begin{cases}s & \text { if } x_{p} \in \mathcal{X}_{\text {para }}^{+}\left(x_{i}\right) \\ 1, & \text { otherwise }\end{cases}
$$

\subsection{Training with $\mathcal{L}_{\mathrm{SSC}}$ and $\mathcal{L}_{\mathrm{CE}}$}

We experiment with various schemes of combining supervision from $\mathcal{L}_{\mathrm{SSC}}$ and $\mathcal{L}_{\mathrm{CE}}$ losses. Specifically, we try - alternate (Algorithm 1), joint, and pretrain-finetune [26] training schemes.

Our alternate training scheme is summarized in Algorithm 1. Specifically, given $N$ total training iterations, we update our model with $\mathcal{L}_{\mathrm{SSC}}$ after every $N_{c e}-1$ updates with $\mathcal{L}_{\mathrm{CE}}$, where $N_{c e}$ is a hyper-parameter. In the joint training scheme, we curate loss-specific batches for $\mathcal{L}_{\mathrm{SSC}}$ and $\mathcal{L}_{\mathrm{CE}}$ but jointly update the model by accumulating the gradients of these two losses. Training alternately or jointly with the two losses simplifies the optimization procedure compared to two-stage training (pretrain-finetune as in [26]) which requires double the hyper-parameters and longer training iterations. Please refer to supplementary for the exact algorithms of joint and pretrain-finetune training schemes. Empirically, we see that alternate training works slightly better than joint-training, and much better than pretrain-finetune training approach. Figure 2 depicts our training strategy (ConClaT).

\subsection{Negative Types and Batch Creation}

SCL operates with multiple negative samples. For a given reference sample $x=(v, q, a) \in \mathcal{D}^{\text {aug }}$, we define a corresponding set of negatives as samples with ground truth different than the reference $x$ :

$$
\mathcal{X}^{-}(x)=\left\{(\bar{v}, \bar{q}, \bar{a}) \in \mathcal{D}^{\text {aug }} \mid \bar{a} \neq a\right\}
$$

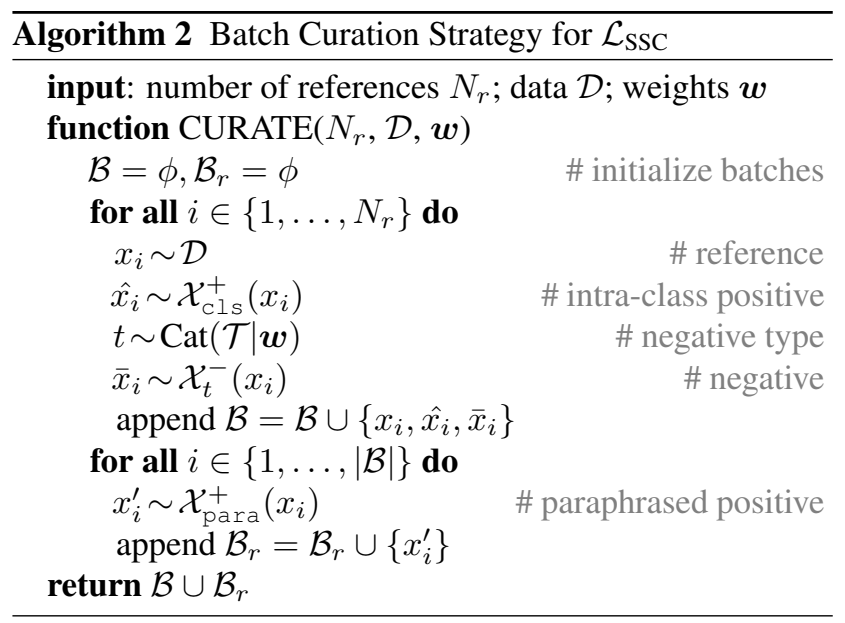

We carefully curate batches for $\mathcal{L}_{\mathrm{SSC}}$ by sampling different types of negatives. We classify a negative sample $\bar{x}=(\bar{v}, \bar{q}, \bar{a}) \in \mathcal{X}^{-}(x)$ into one of three negative categories defined below.

- Image Negatives, $\mathcal{X}_{\text {img }}^{-}(x)$ : Image negatives are samples that have the same image $(v=\bar{v})$ as the reference $(x)$ but different answer. Since VQA dataset has multiple questions $(\sim 5.4)$ per image, finding image negatives is trivial.

- Question Negatives, $\mathcal{X}_{\text {que }}^{-}(x)$ : Question negatives are samples that have questions similar to the reference but different answer. We measure the similarity between the questions by computing their cosine distance in the vector space of the Sentence-BERT [42] model, i.e. $\operatorname{sim}(q, \bar{q})>\epsilon$, where $\epsilon$ is a similarity threshold.

- Random Negatives, $\mathcal{X}_{\text {rand }}^{-}(x)$ : Random negatives are samples that do not fall under either Image or Question negative categories i.e. any image and question pair that has a different answer than the reference.

We hypothesize that discriminating between joint $\mathrm{V}+\mathrm{L}$ representations of above negatives and the reference would lead to more robust $\mathrm{V}+\mathrm{L}$ representations as it requires the model to preserve relevant information from both modalities in the learnt representation. Negative samples belonging to each of the above types are depicted in Figure 2(b).

Batch Curation. To create mini-batches for $\mathcal{L}_{\mathrm{SSC}}$, as described in Algorithm 2, we start by filling our batch with triplets of reference $x_{i}$, a intra-class positive $\hat{x_{i}}$ and a negative sample $\bar{x}_{i}$ of type $t$. The negative type $t$ is sampled from a categorical distribution $\operatorname{Cat}(\mathcal{T} \mid \boldsymbol{w})$ where $\boldsymbol{w}=$ $\left(w_{\text {img }}, w_{\text {que }}, w_{\text {rand }}\right)$ are the probability weights of selecting different types of negatives defined by $\mathcal{T}=$ \{que, img, rand $\}$. This procedure is repeated for specified number of times $N_{r}$ to create a batch $\mathcal{B}$. Finally, for every sample 
in $\mathcal{B}$ we add a corresponding paraphrased positive $x_{i}^{\prime}$ sample. For $\mathcal{L}_{\mathrm{CE}}$, we sample mini-batches randomly from the dataset $\mathcal{D}^{\text {aug }}$.

Importance of Scaling Factor. VQA Dataset has a skewed distribution of answer labels and since we sample references for SCL minibatch independently of each other (see Algorithm 2) quite often we end up with multiple intra-class positives but only a single paraphrased positive for given a reference in a minibatch. To balance this trade-off we choose to scale the loss corresponding to paraphrased positive sample from the intra-class positive samples. We call this loss Scaled Supervised Contrastive Loss $\left(\mathcal{L}_{\mathrm{SSC}}\right)$.

\section{Experiments}

\subsection{Datasets and Metrics}

We use the VQA v2.0 [18] and the VQARephrasings [45] datasets for experiments. VQA contains nearly $443 \mathrm{~K}$ train, $214 \mathrm{~K}$ val and $453 \mathrm{~K}$ test instances. VQA-Rephrasings was collected to evaluate the robustness of VQA models towards human rephrased questions. Specifically, the authors collected 3 human-provided rephrasings for 40k image-question pairs from the VQA v2.0 validation dataset.

Shah et al. [45] also introduced Consensus Score (CS) as an evaluation metric to quantify the agreement of VQA models across multiple rephrasings of the same question. Amongst all subsets of paraphrased questions of size $\mathrm{k}$, the consensus score $\mathbf{C S}(\mathbf{k})$ measures the fraction of subsets in which all the answers have non-zero VQA-Score. For a set of paraphrases $Q$, the consensus score $\mathbf{C S}(\mathbf{k})$ is defined as:

$$
\begin{gathered}
\mathbf{C S}(\mathbf{k})=\sum_{Q^{\prime} \subset Q,\left|Q^{\prime}\right|=k} \frac{\mathcal{S}\left(Q^{\prime}\right)}{{ }^{n} C_{k}} \\
\mathcal{S}\left(Q^{\prime}\right)= \begin{cases}1 & \text { if } \forall q \in Q^{\prime}, \quad \operatorname{VQA}-\operatorname{Score}(q)>0, \\
0 & \text { else }\end{cases}
\end{gathered}
$$

Where ${ }^{n} C_{k}$ is number of subsets of size $k$ sampled from a set of size $n$. $\mathbf{C S}(\mathbf{k})$ is zero for a group of questions $Q$ when the model answers at least $k$ questions correctly.

When reporting results on the val split and VQARephrasings, we train on the VQA 2.0 train split and when reporting results on the VQA 2.0 test-dev and test-std we train on both VQA 2.0 train and val splits. The VQA Rephrasings dataset [45] is never used for training and used only for evaluation.

\subsection{Baselines and Training Details}

VQA Model. For $f$, we use a multimodal transformer (MMT) inspired from [13], with 6 layers and 768-dim embeddings. It takes as input two different modalities. The question tokens are encoded using a pre-trained three layer BERT [14] encoder which is fine-tuned along with the multimodal transformer. Object regions are encoded by extracting features from a frozen ResNeXT-152 [55] based Faster R-CNN model [43]. The projection module $g$ consists of two linear layers and a L-2 normalization function. We choose MMT as representative of current SoTA models $[23,32,13,30,17]$ in VQA that rely heavily on some form of multi-modal transformer architecture. Also note that our approach (ConClaT) is agnostic to the choice of the model.

Question Paraphrases using VQG. Apart from training with question paraphrases generated via Back-translation, we also experiment with generating question paraphrases using the VQG module from [45]. We input the VQG module with 88 random noise vectors to keep the generation comparable with Back-translation approach. For filtering, we use the gating mechanism used by the authors and sentence similarity score of $\geq 0.85$ and keep a maximum of 3 unique rephrasings for each question.

Training Details. We train our models using Adam optimizer [28] with a linear warmup and with a learning rate of 1e-4 and a staircase learning rate schedule, where we multiply the learning rate by 0.2 at $10.6 \mathrm{~K}$ and at $15 \mathrm{~K}$ iterations. We train for 5 epochs of train + augmented dataset on 4 NVIDIA Titan XP GPUs and use a batch-size of 420 when using $\mathcal{L}_{\mathrm{SSC}}$ and $\mathcal{L}_{\mathrm{CE}}$ both and 210 otherwise. We put remaining hyperparameters in the supplementary.

Existing state-of-the-art methods. Previous work [45] in VQA-Rephrasings trained a VQG model using a cycleconsistent training scheme along with the VQA model. The approach involved generating questions by a VQG model such that the answer for the original and the generated question are consistent with each other. For their experiments, they build on top of Pythia [24] and BAN [6] as base VQA models. We treat these approaches as baselines for our experiments.

\section{Results}

In this section, we carefully ablate each component of ConClaT, and also compare results with previous methods (Pythia+CC, BAN+CC) from [45]. We report the Consensus Score $(\mathbf{C S}(\mathbf{k}))$ for $k=3,4$ on VQA-Rephrasings [45] and VQA Accuracy on VQA 2.0 [18] datasets. We omit CS(1) and $\mathbf{C S}(2)$ for brevity, and provide them in the supplementary.

\subsection{ConClaT}

Our baseline architecture MMT without any additional data (Table 2, Row 5) and trained using cross-entropy $\left(\mathcal{L}_{\mathrm{CE}}\right)$ outperforms previous best (BAN+CC, Table 2, Row 4) by $+3.64 \%$ on $\mathbf{C S}(4)$ while being $-0.31 \%$ worse on VQA 2.0 


\begin{tabular}{ccccccccc}
\hline & Model & Loss $($ es $)$ & Scaling & N-Type & Train Scheme & CS(3) & CS(4) & $\begin{array}{c}\text { VQA } \\
\text { val }\end{array}$ \\
\hline 1 & MMT & $\mathcal{L}_{\mathrm{CE}}$ & - & - & - & 55.53 & 52.36 & 66.31 \\
2 & $\mathrm{MMT}$ & $\mathcal{L}_{\mathrm{SSC}} \& \mathcal{L}_{\mathrm{CE}}$ & $\checkmark$ & $\mathrm{R}$ & Alternate & 56.53 & 53.42 & 66.62 \\
3 & $\mathrm{MMT}$ & $\mathcal{L}_{\mathrm{SC}} \& \mathcal{L}_{\mathrm{CE}}$ & $\checkmark$ & $\mathrm{RQ}$ & Alternate & 56.88 & 53.77 & 66.97 \\
4 & $\mathrm{MMT}$ & $\mathcal{L}_{\mathrm{SSC}} \& \mathcal{L}_{\mathrm{CE}}$ & $\checkmark$ & $\mathrm{RI}$ & Alternate & 56.91 & 53.79 & 66.93 \\
5 & $\mathrm{MMT}$ & $\mathcal{L}_{\mathrm{SSC}} \& \mathcal{L}_{\mathrm{CE}}$ & $\checkmark$ & $2 \mathrm{I}$ & Alternate & 57.00 & 53.90 & 66.95 \\
6 & $\mathrm{MMT}$ & $\mathcal{L}_{\mathrm{SSC}} \& \mathcal{L}_{\mathrm{CE}}$ & $\checkmark$ & $\mathrm{RQI}$ & Alternate & $\mathbf{5 7 . 0 8}$ & $\mathbf{5 3 . 9 9}$ & $\mathbf{6 6 . 9 8}$ \\
\hline 7 & $\mathrm{MMT}$ & $\mathcal{L}_{\mathrm{SC}} \& \mathcal{L}_{\mathrm{CE}}$ & $\boldsymbol{X}$ & $\mathrm{RQI}$ & Alternate & 56.49 & 53.36 & 66.60 \\
8 & $\mathrm{MMT}$ & $\mathcal{L}_{\mathrm{SSC}} \& \mathcal{L}_{\mathrm{CE}}$ & Dynamic $($ Eq. 12) & $\mathrm{RQI}$ & Alternate & 57.01 & 53.92 & 66.95 \\
\hline 9 & $\mathrm{MMT}$ & $\mathcal{L}_{\mathrm{SSC}} \& \mathcal{L}_{\mathrm{CE}}$ & $\boldsymbol{J}$ & $\mathrm{RQI}$ & Joint & 56.59 & 53.63 & 66.23 \\
10 & $\mathrm{MMT}$ & $\mathcal{L}_{\mathrm{SSC}} \rightarrow \mathcal{L}_{\mathrm{CE}}[26]$ & $\boldsymbol{x}$ & $\mathrm{RQI}$ & Pretrain-Finetune & 52.63 & 49.20 & 64.21 \\
\hline 11 & $\mathrm{MMT}$ & $\mathcal{L}_{\mathrm{DMT}}[60] \& \mathcal{L}_{\mathrm{CE}}$ & $\boldsymbol{x}$ & $\mathrm{RQI}$ & Alternate & 56.23 & 53.10 & 66.59 \\
\hline
\end{tabular}

Table 1: Ablations Study. Scaling denotes whether scaling factor $\alpha$ (defined in Eq. 9 or Eq. 12) was used. N-Type defines the type of negatives used from Image (I), Question (Q) and Random (R). All experiments are run with Back-translation data.

\begin{tabular}{|c|c|c|c|c|c|c|c|}
\hline \multirow{2}{*}{\multicolumn{2}{|c|}{ Model }} & \multirow[b]{2}{*}{ DA } & \multicolumn{2}{|c|}{ Consensus Scores } & \multicolumn{3}{|c|}{ VQA Scores } \\
\hline & & & $\mathrm{CS}(3)$ & CS(4) & val & test-dev & test-std \\
\hline 1 & Pythia [24] & - & 45.94 & 39.49 & 65.78 & 68.43 & - \\
\hline 2 & BAN [27] & - & 47.45 & 39.87 & 66.04 & 69.64 & - \\
\hline 3 & Pythia + CC [45] & - & 50.92 & 44.30 & 66.03 & 68.88 & - \\
\hline 4 & $\mathrm{BAN}+\mathrm{CC}[45]$ & - & 51.76 & 48.18 & 66.77 & 69.87 & - \\
\hline 5 & MMT & - & 55.10 & 51.82 & 66.46 & - & - \\
\hline 6 & MMT & VQG [45] & 54.92 & 51.85 & 64.50 & - & - \\
\hline 7 & $\mathrm{MMT}+\mathrm{ConClaT}$ & VQG [45] & 55.33 & 52.31 & 64.74 & - & - \\
\hline 8 & MMT & BT & 55.53 & 52.36 & 66.31 & 69.51 & 69.22 \\
\hline 9 & MMT + ConClaT & BT & 57.08 & $\mathbf{5 3 . 9 9}$ & 66.98 & 69.80 & 70.00 \\
\hline
\end{tabular}

Table 2: ConClaT vs existing methods / baselines on VQARephrasings and VQA 2.0. DA denotes the source of augmented data from either Back Translation (BT) or Visual Question Generation (VQG). For test-dev and test-std, we train our model on train+val set of VQA 2.0.

validation. Training MMT with Back-translated data (Table 2, Row 8) using only $\mathcal{L}_{\mathrm{CE}}$ further improves $\mathbf{C S}(\mathbf{4})$ by $+0.54 \%$ while slightly degrading performance on VQA 2.0 by $-0.15 \%$, we treat this as our new baseline.

We find that alternate training (ConClaT) with $\mathcal{L}_{\mathrm{SSC}}$ and $\mathcal{L}_{\mathrm{CE}}$ (Table 2, Row 9) improves CS(4) by $+1.63 \%$ and VQA Accuracy by $+0.67 \%$ on validation. ConClaT outperforms previous state-of-the-art approach $\mathrm{BAN}+\mathrm{CC}$ by $+5.81 \%$ on CS(4) while performing competitively on VQA 2.0 validation $(+0.22 \%)$ and test-dev (-0.07\%) splits. We present this as our main result, which shows that training with both the losses together leads to models that are accurate (higher VQA score) and robust (higher Consensus score).

ConClaT with VQG data. We also experiment by augmenting the data generated from VQG model of [45]. Similar to Back-translation data, we find that using ConClaT (Table 2, Row 7) leads to $+0.46 \%$ and $+0.24 \%$ gains on CS(4) and VQA 2.0 validation over the baseline (Table 2,
Row 8). We attribute the relatively smaller gains from VQG data to the lower quality and lesser quantity of paraphrases generated by the VQG module. We discuss more about the quality of generated data in Supplementary Section 5.

\subsection{Ablations}

Training schemes. We try three different ways of combining $\mathcal{L}_{\mathrm{CE}}$ and $\mathcal{L}_{\mathrm{SSC}}$ losses. Training alternately performs the best (Table 1, Row 6), whereas training jointly performs worse by $-0.36 \%$ and $-0.75 \%$ on $\mathbf{C S ( 4 )}$ and VQA validation accuracy respectively (Table 1, Row 9). Following the approach taken in [26], we try pre-training the model with $\mathcal{L}_{\mathrm{SSC}}$ and then finetuning it on $\mathcal{L}_{\mathrm{CE}}$ (Table 1, Row 10) and we find this to perform the worst with $-4.79 \%$ and $-2.77 \%$ in CS(4) and VQA validation accuracy respectively .

Contrastive vs Triplet Losses. Previous works have explored the use of triplet losses [60,39] for learning robust VQA models. Specifically, we experiment by replacing our $\mathcal{L}_{\text {SSC }}$ with Dynamic-margin Triplet loss $\left(\mathcal{L}_{\text {DMT }}\right)$ proposed in [60] for mitigating the tendency of VQA models to ignore the image and rely solely on question for answering (also known as knowledge-inertia) . It is also worth noting that $\mathcal{L}_{\mathrm{DMT}}$ is an improved version of the vanilla triplet loss used in [39]. We find that ConClaT outperforms this ablation (Table 1, Row 11) by $+0.89 \%$ and $+0.39 \% \mathbf{C S}(\mathbf{4})$ and VQA validation accuracy respectively.

Scaling in $\mathcal{L}_{\text {SSC }}$. We see improvement on both VQA validation $(+0.56 \%)$ and $\mathbf{C S}(4)(+0.35 \%)$ when using our proposed variant Scaled Supervised Contrastive Loss $\left(\mathcal{L}_{\mathrm{SSC}}\right)$ when compared to using unscaled $\mathcal{L}_{\mathrm{SC}}$ (Table 1, Rows 6, 7). Beyond the constant scaling factor defined in Eq. 9, we also experimented with using a dynamic scaling factor de- 

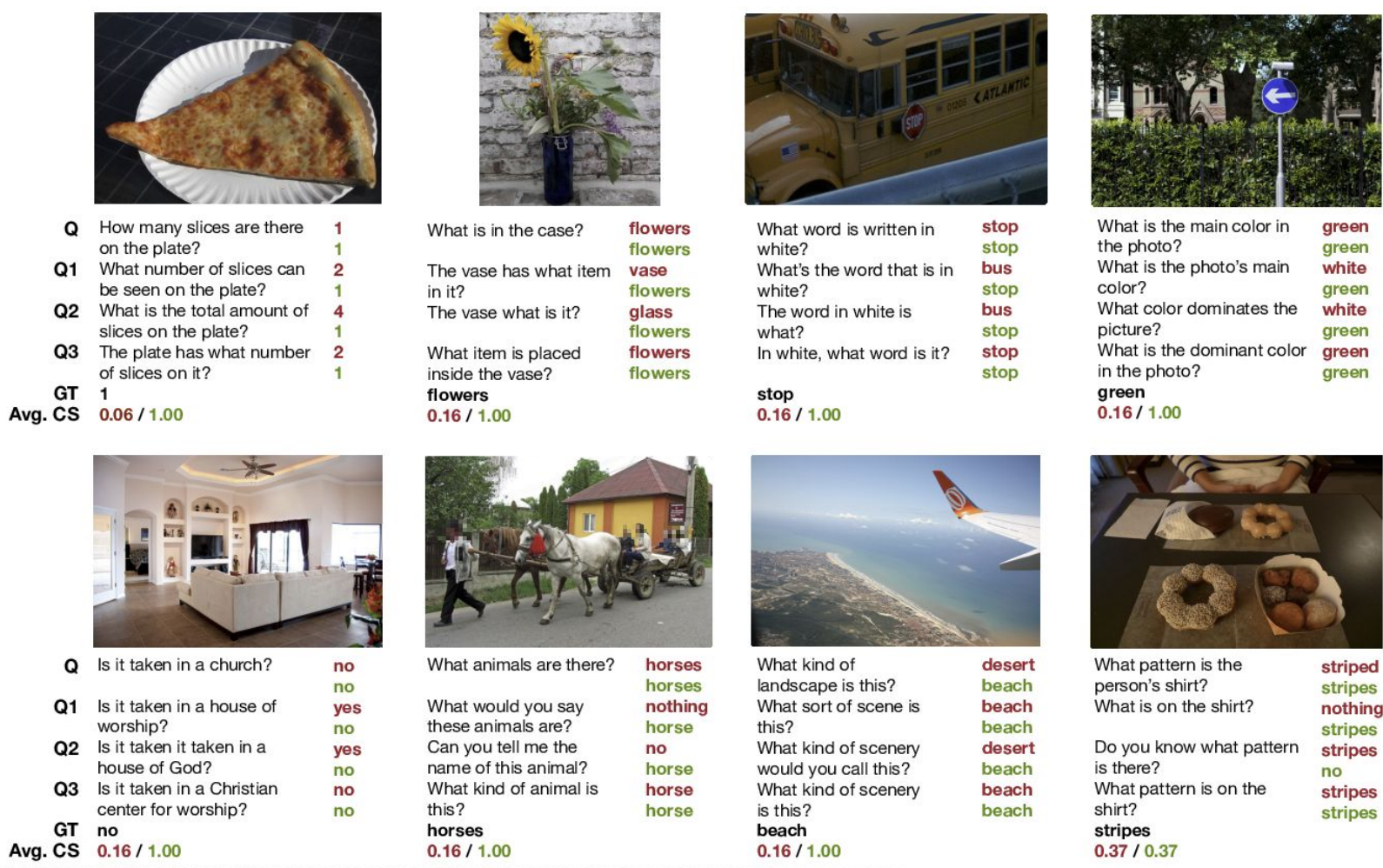

What is the main color in green the photo? green What is the photo's main white color? What color dominates the white picture? green What is the dominant color green in the photo? green green stop
$0.16 / 1.00$

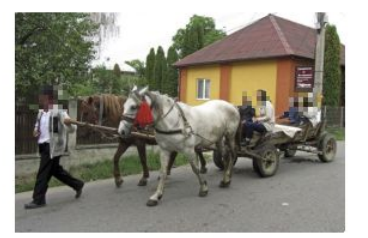

What animals are there? horses

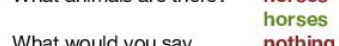

What would you say nothing these animals are? hors Can you tell me the no name of this animal? horse What kind of animal is horse this?

horses $0.16 / 1.00$
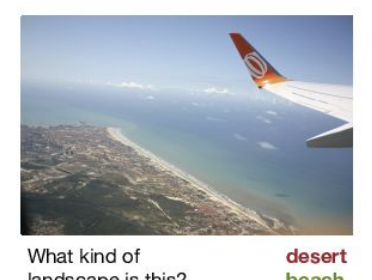

landscape is this? What sort of scene is beach this? beach

What kind of scenery would you call this? What kind of scenery beach is this?

beach

$0.16 / 1.00$

$0.16 / 1.00$

Baseline

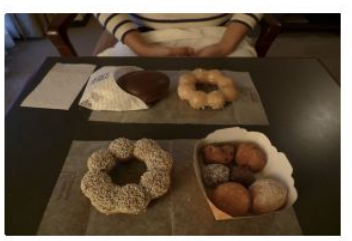

What pattern is the striped person's shirt? What is on the shirt? What is on the shirt? nothing Do you know what pattern stripes is there? no What pattern is on the stripes shirt? stripes stripes
$0.37 / 0.37$

Figure 3: Qualitative Examples. Predictions of ConClaT (Table 1, Row 1) and our baseline (Table 1, Row 6) on several image-question pairs and their corresponding rephrased questions. Average Consensus Scores $(\mathrm{k}=1-4)$ are also shown at the bottom (higher the better).

fined as follows:

$$
\alpha_{i p}= \begin{cases}s . \Phi\left(\boldsymbol{z}_{i} \cdot \boldsymbol{z}_{p}\right) & \text { if } x_{p} \in \mathcal{X}_{\text {para }}^{+}\left(x_{i}\right) \\ \Phi\left(\boldsymbol{z}_{i} \cdot \boldsymbol{z}_{p}\right), & \text { otherwise }\end{cases}
$$

Where $\Phi(\boldsymbol{u}, \boldsymbol{v})=1-\boldsymbol{u}^{\top} \boldsymbol{v} /\|\boldsymbol{u}\|\|\boldsymbol{v}\|$ computes the cosine distance between $\boldsymbol{u}$ and $\boldsymbol{v}$. We did not find significant improvements using dynamic scaling (Table 1, Row 8).

Negative Sampling Strategy. Furthermore, we find that our proposed negative sampling strategy (Algorithm 2) where we carefully curate batches for $\mathcal{L}_{\text {SSC }}$ loss (Table 1 , Row 6) helps improve CS(4) $(+0.57 \%)$ and VQA accuracy $(+0.36 \%)$ over random-sampling (Table 1, Row 2$)$. We find that adding either que-type negatives (Table 1, Row 3) or img-type negatives(Table 1, Row 4) lead to gains in CS(4) and VQA validation accuracy. Using only img-type and que-type negatives (Table 1, Row 5) leads to significant gains, showing that use of both the types is crucial.

\subsection{Qualitative Analysis}

We qualitatively visualize few samples in Figure 3. We compare our final approach (Table 1, Row 6) with our baseline (Table 1, Row 1). As evident from samples, ConClaT improves the consistency in answers across the rephrasings.
$(2,2)$ shows an interesting example where ConClaT yields a singular answer for one question paraphrase and produces the original plural answer for other paraphrased question. In $(2,3)$, baseline incorrectly answers the original VQA question but correctly answers some of the rephrasings whereas our approach gets all the questions right. $(2,4)$ illustrates a failure case where both the approaches fail to answer all the paraphrased questions correctly.

\section{Conclusion}

To summarize, we have three main contributions. First, we propose a novel training paradigm (ConClaT) that optimizes contrastive and cross-entropy losses to learn joint vision and language representations that are robust to question paraphrases. Minimizing the contrastive loss encourages representations to be robust to linguistic variations in questions while the cross-entropy loss preserves the discriminative power of the representations for answer classification. Second, we introduce Scaled Supervised Contrastive Loss $\left(\mathcal{L}_{\mathrm{SSC}}\right)$, that assigns higher weight to positive samples associated with question paraphrases over samples that just have the same answer boosting the performance further. Finally, we propose a negative sampling strategy to curate 
loss-specific batches which improves performance over random sampling strategy. Compared to previous approaches, VQA models trained with ConClaT achieve higher consistency scores on the VQA-Rephrasings dataset as well as higher VQA accuracy on the VQA 2.0 dataset across a variety of data augmentation strategies. We also qualitatively demonstrate that our approach yields correct and consistent answers for VQA questions and their rephrasings.

\section{Acknowledgements}

We thank Abhishek Das, Prithvijit Chattopadhyay and Arjun Majumdar for their feedback. The Georgia Tech effort was supported in part by NSF, AFRL, DARPA, ONR YIPs, ARO PECASE, Amazon. The views and conclusions contained herein are those of the authors and should not be interpreted as necessarily representing the official policies or endorsements, either expressed or implied, of the U.S. Government, or any sponsor.

\section{References}

[1] E. Abbasnejad, D. Teney, A. Parvaneh, J. Shi, and A. van den Hengel. Counterfactual vision and language learning. In 2020 IEEE/CVF Conference on Computer Vision and Pattern Recognition (CVPR), pages 10041-10051, 2020. 2

[2] Vedika Agarwal, Rakshith Shetty, and Mario Fritz. Towards causal vqa: Revealing and reducing spurious correlations by invariant and covariant semantic editing, 2020. 2

[3] Aishwarya Agrawal, Dhruv Batra, Devi Parikh, and Aniruddha Kembhavi. Don't just assume; look and answer: Overcoming priors for visual question answering, 2017. 2

[4] Aishwarya Agrawal, Aniruddha Kembhavi, Dhruv Batra, and Devi Parikh. C-vqa: A compositional split of the visual question answering (vqa) v1.0 dataset, 2017. 2

[5] Aishwarya Agrawal, Jiasen Lu, Stanislaw Antol, Margaret Mitchell, C. Lawrence Zitnick, Dhruv Batra, and Devi Parikh. Vqa: Visual question answering, 2015. 3

[6] Peter Anderson, Xiaodong He, Chris Buehler, Damien Teney, Mark Johnson, Stephen Gould, and Lei Zhang. Bottom-up and top-down attention for image captioning and visual question answering, 2017. 2, 3, 6

[7] Jacob Andreas, Marcus Rohrbach, Trevor Darrell, and Dan Klein. Neural module networks, 2015. 2

[8] Hao-Yun Chen, Pei-Hsin Wang, Chun-Hao Liu, Shih-Chieh Chang, Jia-Yu Pan, Yu-Ting Chen, Wei Wei, and Da-Cheng Juan. Complement objective training, 2019. 2

[9] Long Chen, Xin Yan, Jun Xiao, Hanwang Zhang, Shiliang $\mathrm{Pu}$, and Yueting Zhuang. Counterfactual samples synthesizing for robust visual question answering, 2020. 2

[10] Ting Chen, Simon Kornblith, Mohammad Norouzi, and Geoffrey Hinton. A simple framework for contrastive learning of visual representations. arXiv preprint arXiv:2002.05709, 2020. 3

[11] Ting Chen, Simon Kornblith, Kevin Swersky, Mohammad Norouzi, and Geoffrey Hinton. Big self-supervised mod- els are strong semi-supervised learners. arXiv preprint arXiv:2006.10029, 2020. 3

[12] Xinlei Chen, Haoqi Fan, Ross Girshick, and Kaiming He. Improved baselines with momentum contrastive learning. arXiv preprint arXiv:2003.04297, 2020. 3

[13] Yen-Chun Chen, Linjie Li, Licheng Yu, Ahmed El Kholy, Faisal Ahmed, Zhe Gan, Yu Cheng, and Jingjing Liu. Uniter: Learning universal image-text representations. arXiv preprint arXiv:1909.11740, 2019. 2, 3, 6

[14] Jacob Devlin, Ming-Wei Chang, Kenton Lee, and Kristina Toutanova. Bert: Pre-training of deep bidirectional transformers for language understanding. arXiv preprint arXiv:1810.04805, 2018. 2, 6

[15] Sergey Edunov, Myle Ott, Michael Auli, and David Grangier. Understanding back-translation at scale, 2018. 2

[16] Akira Fukui, Dong Huk Park, Daylen Yang, Anna Rohrbach, Trevor Darrell, and Marcus Rohrbach. Multimodal compact bilinear pooling for visual question answering and visual grounding. arXiv preprint arXiv:1606.01847, 2016. 2

[17] Akira Fukui, Dong Huk Park, Daylen Yang, Anna Rohrbach, Trevor Darrell, and Marcus Rohrbach. Multimodal compact bilinear pooling for visual question answering and visual grounding, 2016. 6

[18] Yash Goyal, Tejas Khot, Douglas Summers-Stay, Dhruv Batra, and Devi Parikh. Making the v in vqa matter: Elevating the role of image understanding in visual question answering, 2016. 2, 3, 6

[19] Ankush Gupta, Arvind Agarwal, Prawaan Singh, and Piyush Rai. A deep generative framework for paraphrase generation. arXiv preprint arXiv:1709.05074, 2017. 2

[20] Tanmay Gupta, Arash Vahdat, Gal Chechik, Xiaodong Yang, Jan Kautz, and Derek Hoiem. Contrastive learning for weakly supervised phrase grounding. 2020. 3

[21] Kaiming He, Haoqi Fan, Yuxin Wu, Saining Xie, and Ross Girshick. Momentum contrast for unsupervised visual representation learning. In Proceedings of the IEEE/CVF Conference on Computer Vision and Pattern Recognition, pages 9729-9738, 2020. 3

[22] Olivier J Hénaff, Aravind Srinivas, Jeffrey De Fauw, Ali Razavi, Carl Doersch, SM Eslami, and Aaron van den Oord. Data-efficient image recognition with contrastive predictive coding. arXiv preprint arXiv:1905.09272, 2019. 3

[23] Huaizu Jiang, I. Misra, Marcus Rohrbach, E. Learned-Miller, and Xinlei Chen. In defense of grid features for visual question answering. 2020 IEEE/CVF Conference on Computer Vision and Pattern Recognition (CVPR), pages 10264 10273, 2020. 2, 6

[24] Yu Jiang, Vivek Natarajan, Xinlei Chen, Marcus Rohrbach, Dhruv Batra, and Devi Parikh. Pythia v0.1: the winning entry to the vqa challenge 2018, 2018. 1, 2, 3, 6, 7

[25] Marcin Junczys-Dowmunt, Roman Grundkiewicz, Tomasz Dwojak, Hieu Hoang, Kenneth Heafield, Tom Neckermann, Frank Seide, Ulrich Germann, Alham Fikri Aji, Nikolay Bogoychev, André F. T. Martins, and Alexandra Birch. Marian: Fast neural machine translation in $\mathrm{C}++$. In Proceedings of ACL 2018, System Demonstrations, pages 116-121, Melbourne, Australia, July 2018. Association for Computational Linguistics. 3, 13 
[26] Prannay Khosla, Piotr Teterwak, Chen Wang, Aaron Sarna, Yonglong Tian, Phillip Isola, A. Maschinot, Ce Liu, and Dilip Krishnan. Supervised contrastive learning. ArXiv, abs/2004.11362, 2020. 2, 3, 4, 5, 7, 15

[27] Jin-Hwa Kim, Jaehyun Jun, and Byoung-Tak Zhang. Bilinear attention networks. In Advances in Neural Information Processing Systems, pages 1564-1574, 2018. 2, 7

[28] Diederik P. Kingma and Jimmy Ba. Adam: A method for stochastic optimization, 2014. 6, 12

[29] Gen Li, Nan Duan, Yuejian Fang, Ming Gong, Daxin Jiang, and Ming Zhou. Unicoder-vl: A universal encoder for vision and language by cross-modal pre-training. In $A A A I$, pages 11336-11344, 2020. 2

[30] Xiujun Li, Xi Yin, Chunyuan Li, Xiaowei Hu, Pengchuan Zhang, Lei Zhang, Lijuan Wang, Houdong Hu, Li Dong, Furu Wei, et al. Oscar: Object-semantics aligned pre-training for vision-language tasks. arXiv preprint arXiv:2004.06165, 2020. 6

[31] Zichao Li, Xin Jiang, Lifeng Shang, and Hang Li. Paraphrase generation with deep reinforcement learning. arXiv preprint arXiv:1711.00279, 2017. 2

[32] Jiasen Lu, Dhruv Batra, Devi Parikh, and Stefan Lee. Vilbert: Pretraining task-agnostic visiolinguistic representations for vision-and-language tasks. In Advances in Neural Information Processing Systems, pages 13-23, 2019. 2, 6

[33] Jiasen Lu, Vedanuj Goswami, Marcus Rohrbach, Devi Parikh, and Stefan Lee. 12-in-1: Multi-task vision and language representation learning. In Proceedings of the IEEE/CVF Conference on Computer Vision and Pattern Recognition, pages 10437-10446, 2020. 2

[34] Jiasen Lu, Jianwei Yang, Dhruv Batra, and Devi Parikh. Hierarchical question-image co-attention for visual question answering, 2016. 2

[35] Jonathan Mallinson, Rico Sennrich, and Mirella Lapata. Paraphrasing revisited with neural machine translation. In Proceedings of the 15th Conference of the European Chapter of the Association for Computational Linguistics: Volume 1, Long Papers, pages 881-893, 2017. 3

[36] A. Oord, Y. Li, and Oriol Vinyals. Representation learning with contrastive predictive coding. ArXiv, abs/1807.03748, 2018. 1,3

[37] Jingjing Pan, Yash Goyal, and Stefan Lee. Questionconditioned counterfactual image generation for vqa, 2019. 2

[38] Adam Paszke, S. Gross, Francisco Massa, A. Lerer, J. Bradbury, G. Chanan, T. Killeen, Z. Lin, N. Gimelshein, L. Antiga, Alban Desmaison, Andreas Köpf, E. Yang, Zach DeVito, Martin Raison, Alykhan Tejani, Sasank Chilamkurthy, B. Steiner, Lu Fang, Junjie Bai, and Soumith Chintala. Pytorch: An imperative style, high-performance deep learning library. ArXiv, abs/1912.01703, 2019. 13

[39] Badri Patro and Vinay P. Namboodiri. Differential attention for visual question answering, 2018. 2, 7

[40] Aaditya Prakash, Sadid A Hasan, Kathy Lee, Vivek Datla, Ashequl Qadir, Joey Liu, and Oladimeji Farri. Neural paraphrase generation with stacked residual lstm networks. arXiv preprint arXiv:1610.03098, 2016. 2
[41] Alec Radford, Jong Wook Kim, Chris Hallacy, Aditya Ramesh, Gabriel Goh, Sandhini Agarwal, Girish Sastry, Amanda Askell, Pamela Mishkin, Jack Clark, Gretchen Krueger, and Ilya Sutskever. Learning transferable visual models from natural language supervision, 2021. 3

[42] Nils Reimers and Iryna Gurevych. Sentence-bert: Sentence embeddings using siamese bert-networks, 2019. 2, 3, 5, 13

[43] Shaoqing Ren, Kaiming He, Ross Girshick, and Jian Sun. Faster r-cnn: Towards real-time object detection with region proposal networks. 2015. 6

[44] Ramprasaath R Selvaraju, Purva Tendulkar, Devi Parikh, Eric Horvitz, Marco Tulio Ribeiro, Besmira Nushi, and Ece Kamar. Squinting at vqa models: Introspecting vqa models with sub-questions. In Proceedings of the IEEE/CVF Conference on Computer Vision and Pattern Recognition, pages 10003-10011, 2020. 2

[45] Meet Shah, Xinlei Chen, Marcus Rohrbach, and Devi Parikh. Cycle-consistency for robust visual question answering. In Proceedings of the IEEE conference on computer vision and pattern recognition, pages 6649-6658, 2019. 1, 2, 6, 7, 13, 14

[46] Weijie Su, Xizhou Zhu, Yue Cao, Bin Li, Lewei Lu, Furu Wei, and Jifeng Dai. Vl-bert: Pre-training of generic visuallinguistic representations. arXiv preprint arXiv:1908.08530, 2019. 2

[47] Hao Tan and Mohit Bansal. Lxmert: Learning crossmodality encoder representations from transformers. arXiv preprint arXiv:1908.07490, 2019. 2

[48] Ruixue Tang, Chao Ma, Wei Emma Zhang, Qi Wu, and Xiaokang Yang. Semantic equivalent adversarial data augmentation for visual question answering. 2020. 1, 2

[49] Damien Teney, Ehsan Abbasnedjad, and Anton van den Hengel. Learning what makes a difference from counterfactual examples and gradient supervision, 2020. 2

[50] Su Wang, Rahul Gupta, Nancy Chang, and Jason Baldridge. A task in a suit and a tie: paraphrase generation with semantic augmentation. In Proceedings of the AAAI Conference on Artificial Intelligence, volume 33, pages 7176-7183, 2019. 2

[51] Spencer Whitehead, Hui Wu, Yi Ren Fung, Heng Ji, Rogerio Feris, and Kate Saenko. Learning from lexical perturbations for consistent visual question answering, 2020. 2

[52] John Wieting, Jonathan Mallinson, and Kevin Gimpel. Learning paraphrastic sentence embeddings from backtranslated bitext. arXiv preprint arXiv:1706.01847, 2017. 3

[53] Thomas Wolf, Lysandre Debut, Victor Sanh, Julien Chaumond, Clement Delangue, Anthony Moi, Pierric Cistac, Tim Rault, Rémi Louf, Morgan Funtowicz, Joe Davison, Sam Shleifer, Patrick von Platen, Clara Ma, Yacine Jernite, Julien $\mathrm{Plu}$, Canwen Xu, Teven Le Scao, Sylvain Gugger, Mariama Drame, Quentin Lhoest, and Alexander M. Rush. Huggingface's transformers: State-of-the-art natural language processing. ArXiv, abs/1910.03771, 2019. 2, 3, 13

[54] Zhirong Wu, Yuanjun Xiong, Stella Yu, and Dahua Lin. Unsupervised feature learning via non-parametric instance-level discrimination. arXiv preprint arXiv:1805.01978, 2018. 3

[55] Saining Xie, Ross Girshick, Piotr Dollar, Zhuowen Tu, and Kaiming He. Aggregated residual transformations for deep 
neural networks. 2017 IEEE Conference on Computer Vision and Pattern Recognition (CVPR), 2017. 6

[56] Zichao Yang, Xiaodong He, Jianfeng Gao, Li Deng, and Alex Smola. Stacked attention networks for image question answering, 2015. 2

[57] Tianhe Yu, Saurabh Kumar, Abhishek Gupta, Sergey Levine, Karol Hausman, and Chelsea Finn. Gradient surgery for multi-task learning, 2020. 12

[58] Zhou Yu, Jun Yu, Chenchao Xiang, Jianping Fan, and Dacheng Tao. Beyond bilinear: Generalized multimodal factorized high-order pooling for visual question answering. IEEE transactions on neural networks and learning systems, 29(12):5947-5959, 2018. 2

[59] Peng Zhang, Yash Goyal, Douglas Summers-Stay, Dhruv Batra, and Devi Parikh. Yin and yang: Balancing and answering binary visual questions, 2015. 2

[60] Yiyi Zhou, Rongrong Ji, Jinsong Su, Xiangming Li, and Xiaoshuai Sun. Free vqa models from knowledge inertia by pairwise inconformity learning. Proceedings of the AAAI Conference on Artificial Intelligence, 33(01):93169323, Jul. 2019. 2, 7, 15 


\section{Appendix}

\section{A. Ablations with Joint Training}

In the joint training experiment (Table 2, Row 8), we use a weighing parameter $(\beta)$ to combine the $\mathcal{L}_{\mathrm{SC}}$ and $\mathcal{L}_{\mathrm{CE}}$ losses. We ablate on the choice of weight $(\beta)$ used, and we represent the overall loss in this experiment as:

$$
\mathcal{L}_{\text {joint }}=\beta \mathcal{L}_{\mathrm{SSC}}+(1-\beta) \mathcal{L}_{\mathrm{CE}}
$$

We also find that the VQA-Accuracy and Consensus Scores hit a sweet-spot at $\beta=0.5$ and we use this configuration as our basline.

\begin{tabular}{llccc}
\hline & Model & $\beta$ & CS(4) & $\begin{array}{c}\text { VQA } \\
\text { val }\end{array}$ \\
\hline 1 & MMT & 0.25 & 52.97 & 66.14 \\
2 & MMT & 0.50 & 53.63 & 66.23 \\
3 & MMT & 0.75 & 48.53 & 61.34 \\
4 & MMT & 0.90 & 40.68 & 51.03 \\
5 & MMT + ConClaT & - & $\mathbf{5 3 . 9 9}$ & $\mathbf{6 6 . 9 8}$ \\
\hline
\end{tabular}

Table A: Ablations on the choice of our hyper-parameter $\beta$ for joint training.

\section{B. Joint and Pretrain-Finetune Training}

As mentioned in Section 4.3 of the manuscript, we respectively provide the training schemes used to jointly optimize in Algorithm 3 and the scheme used to pretrainfinetune in Algorithm 4 with the $\mathcal{L}_{\mathrm{SSC}}$ and $\mathcal{L}_{\mathrm{CE}}$ losses.

\section{Gradient Surgery of $\mathcal{L}_{\mathrm{SSC}}$ and $\mathcal{L}_{\mathrm{CE}}$}

To know whether the gradients of both the losses $\left(\mathcal{L}_{\mathrm{SSC}}\right.$ and $\mathcal{L}_{\mathrm{CE}}$ ) are aligned with each other during training, we follow the gradient surgery setup of [57] for multi-task learning. During joint-training, we take the dot-products of gradients from both the losses and plot them to see how well they are aligned i.e. whether the dot product is positive or negative. In Figure A we plot the un-normalized dot product between the gradients corresponding to $\mathcal{L}_{\mathrm{CE}}$ and $\mathcal{L}_{\mathrm{SSC}}$

\footnotetext{
*Correspondence to ysh. kant@gmail. com
}
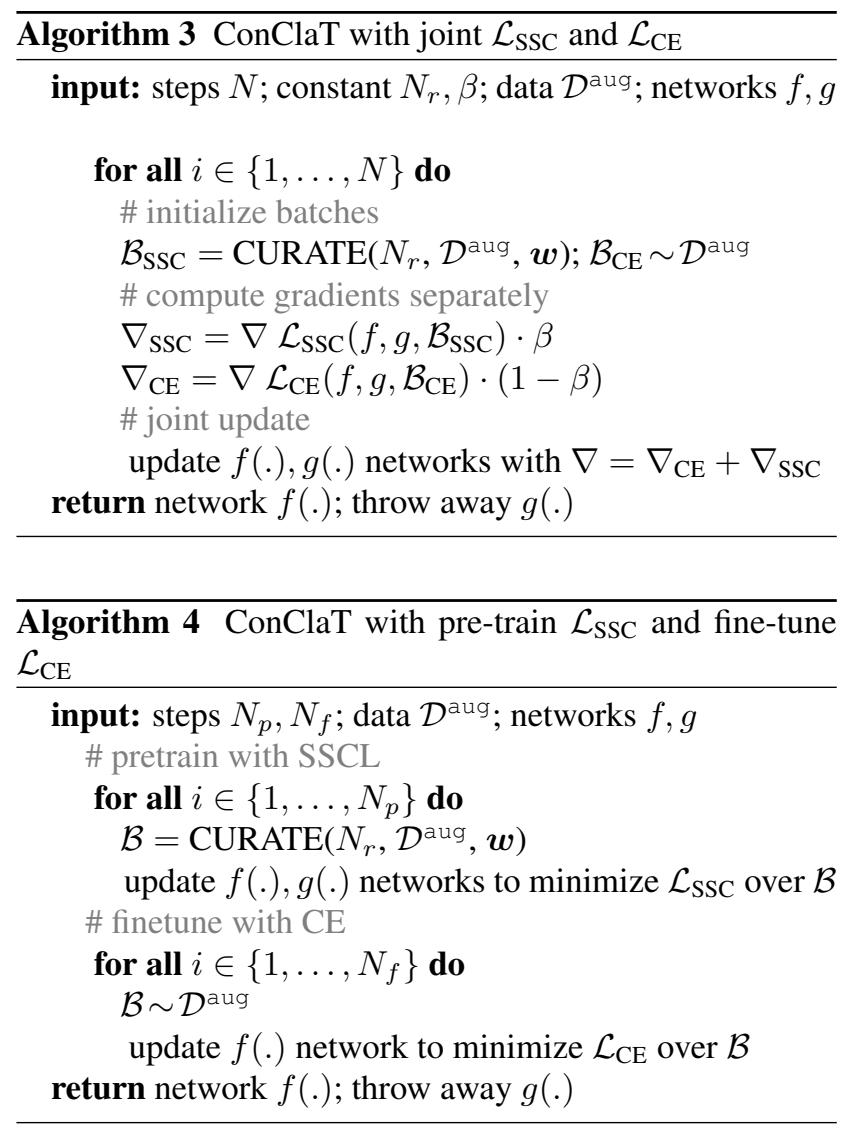

losses. We find that except for initial few steps the gradients of both the losses are aligned (dot product is positive) and thus the updates are complementary with respect to each other.

\section{Training}

Hyperparameters. All the models have $\sim 100 \mathrm{M}$ trainable parameters. We train our models using Adam optimizer [28] with a linear warmup and with a learning rate of 1e-4 and a staircase learning rate schedule, where we multiply the learning rate by 0.2 at $10.6 \mathrm{~K}$ and at $15 \mathrm{~K}$ iterations. We train for 5 epochs of augmented train dataset $\mathcal{D}^{a u g}$ on 4 NVIDIA Titan XP GPUs and use a batch-size of 420 when using $\mathcal{L}_{\mathrm{SSC}}$ and $\mathcal{L}_{\mathrm{CE}}$ both and 210 otherwise. We use Py- 
Table B: Hyperparameter choices for models.

\begin{tabular}{clcclc}
\hline$\#$ & Hyperparameters & Value & $\#$ & Hyperparameters & Value \\
\hline 1 & Maximum question tokens & 23 & 2 & Maximum object tokens & 101 \\
3 & $\mathcal{L}_{\text {CE }}: \mathcal{L}_{\text {SSC }}$ iterations ratio & $3: 1$ & 4 & Number of TextBert layers & 3 \\
5 & Embedding size & 768 & 6 & Number of Multimodal layers & 6 \\
7 & Multimodal layer intermediate size & 3072 & 8 & Number of attention heads & 12 \\
9 & Negative type weights $(\boldsymbol{w})$ & $(0.25,0.25,0.5)$ & 10 & Multimodal layer dropout & 0.1 \\
11 & Similarity Threshold $(\epsilon)$ & 0.95 & 12 & Optimizer & Adam \\
13 & Batch size & $210 / 420$ & 14 & Base Learning rate & $2 \mathrm{e}-4$ \\
15 & Warm-up learning rate factor & 0.1 & 16 & Warm-up iterations & 4266 \\
17 & Vocabulary size & 3129 & 18 & Gradient clipping $($ L-2 Norm) & 0.25 \\
19 & Number of epochs & $5 / 20$ & 20 & Learning rate decay & 0.2 \\
21 & Learning rate decay steps & 10665,14931 & 22 & Number of iterations & 25000 \\
23 & Projection Dimension $\left(\mathcal{R}^{d_{z}}\right)$ & 128 & 24 & Scaling Factor $(s)$ & 20 \\
25 & $N_{c e}$ & 4 & 26 & $N_{r}$ & 70 \\
\hline
\end{tabular}

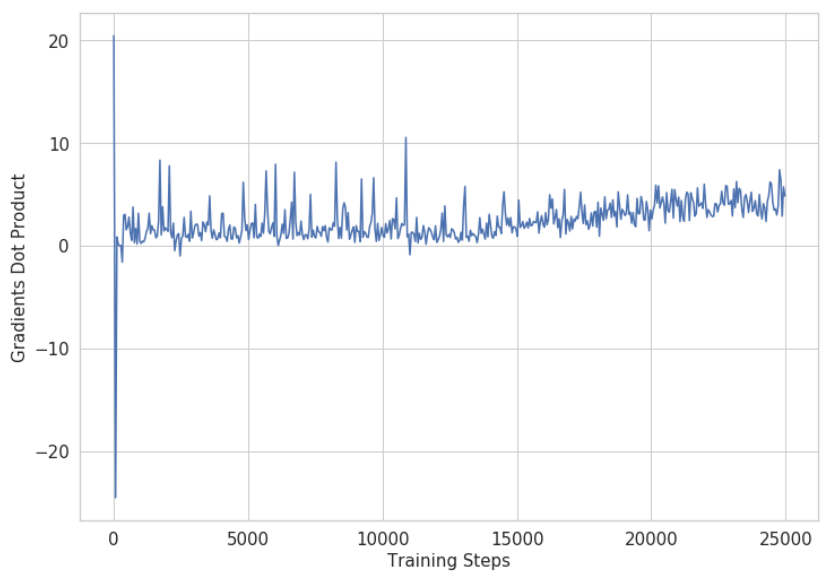

Figure A: Gradient Alignment between the $\mathcal{L}_{\mathrm{SSC}}$ and $\mathcal{L}_{\mathrm{CE}}$ losses. The dot-product is positive indicating that the gradients from the two losses are aligned.

Torch [38] for all the experiments. Hyperparameters are summarized in Table B.

\section{E. Frequently Asked Questions}

Why use different sampling rates for different negative types?

The different types of negatives - same-image-differentquestion (img) and same-question-different-image (que) - encourages the model to be sensitive to both modalities. We use different sampling weights to emphasize more on these two types of negatives over the ones which just have different answers. We obtain the weights (Table B, Row 9) through hyper-parameter tuning on the validation set.

Why should questions dealing with different concepts but same answer (e.g., questions in Fig 2b, "Is the dog atop a sofa?" and "Is there broccoli in the picture?") have similar representations?

We clarify that we do not impose any supervision at the level of MMT layers but only at the penultimate layer before answer prediction. Hence, the model is able to perform different reasoning steps (needed to process entirely different visual/textual inputs) for arriving at the same final answer.

\section{F. Augmented Data}

Back-translation: We use 88 different MarianNMT [25] Back-translation model pairs released by Hugging Face [53] to generate question paraphrases. We use SentenceBERT [42] to filter out paraphrases that cosine similarity of $\geq 0.95$ with the original question and choose three unique paraphrases randomly from the filtered set. After filtering duplicates we end up with 2.89 paraphrases per original question on average.

VQG: We use the VQG model introduced by previous work [45] that takes as input the image and answer to generate a paraphrased question. We input the VQG module with 88 random noise vectors to keep the generation comparable with Back-translation approach. For filtering, we use the gating mechanism used by the authors and sentence similarity score of $\geq 0.85$ and keep a maximum of 3 unique rephrasings for each question. Since, VQG produces fewer unique rephrasings per question than Back-translation, we used a lower similarity threshold. After filtering duplicates we end up with only 0.96 paraphrases per original question on average, far fewer than Back-translation. Qualitatively, we find the VQG paraphrases worse when compared against Back-translated ones.

Evaluation: During training, we evaluate our models using the Back-translated rephrasings on a subset of questions from validation set which do not overlap with VQA- 
Rephrasings [45] dataset.

\section{G. Code and Result Files}

We share the code for running the baseline and the best experiments (Table 1, Rows 5, 9). Please find the released code at: https://www.github.com/yashkant/concat-vqa

\section{H. Full Ablations}

For brevity and conciseness, we omitted $\mathbf{C S}(\mathbf{1})$ and CS(2) scores in the main ablation table, we provide the these scores in Table B.

\section{Qualitative Samples}

Figures B, C, D, E show many more qualitative samples comparing the baseline and ConClaT. We visualize the data generated via Back-translation and mined triplets in Figures F, G, H. 


\begin{tabular}{ccccccccccc}
\hline & Model & Loss $(\mathbf{e s})$ & Scaling & N-Type & Train Scheme & CS(1) & CS(2) & CS(3) & CS(4) & $\begin{array}{c}\text { VQA } \\
\text { val }\end{array}$ \\
\hline 1 & $\mathrm{MMT}$ & $\mathcal{L}_{\mathrm{CE}}$ & - & - & - & 67.58 & 60.04 & 55.53 & 52.36 & 66.31 \\
2 & $\mathrm{MMT}$ & $\mathcal{L}_{\mathrm{SSC}} \& \mathcal{L}_{\mathrm{CE}}$ & $\checkmark$ & $\mathrm{R}$ & Alternate & 68.19 & 60.92 & 56.53 & 53.42 & 66.62 \\
3 & $\mathrm{MMT}$ & $\mathcal{L}_{\mathrm{SC}} \& \mathcal{L}_{\mathrm{CE}}$ & $\checkmark$ & $\mathrm{RQ}$ & Alternate & 68.41 & 61.24 & 56.88 & 53.77 & 66.97 \\
4 & $\mathrm{MMT}$ & $\mathcal{L}_{\mathrm{SSC}} \& \mathcal{L}_{\mathrm{CE}}$ & $\checkmark$ & $\mathrm{RI}$ & Alternate & 68.47 & 61.28 & 56.91 & 53.79 & 66.93 \\
5 & $\mathrm{MMT}$ & $\mathcal{L}_{\mathrm{SSC}} \& \mathcal{L}_{\mathrm{CE}}$ & $\checkmark$ & $\mathrm{QI}$ & Alternate & 68.65 & 61.40 & 57.00 & 53.90 & 66.95 \\
6 & $\mathrm{MMT}$ & $\mathcal{L}_{\mathrm{SSC}} \& \mathcal{L}_{\mathrm{CE}}$ & $\checkmark$ & $\mathrm{RQI}$ & Alternate & $\mathbf{6 8 . 6 2}$ & $\mathbf{6 1 . 4 2}$ & $\mathbf{5 7 . 0 8}$ & $\mathbf{5 3 . 9 9}$ & $\mathbf{6 6 . 9 8}$ \\
\hline 7 & $\mathrm{MMT}$ & $\mathcal{L}_{\mathrm{SC}} \& \mathcal{L}_{\mathrm{CE}}$ & $\boldsymbol{X}$ & $\mathrm{R} Q \mathrm{I}$ & Alternate & 68.20 & 60.90 & 56.49 & 53.36 & 66.60 \\
8 & $\mathrm{MMT}$ & $\mathcal{L}_{\mathrm{SSC}} \& \mathcal{L}_{\mathrm{CE}}$ & $\mathrm{Dynnmic}$ & $\mathrm{R} Q \mathrm{I}$ & Alternate & 68.60 & 61.38 & 57.01 & 53.92 & 66.95 \\
\hline 9 & $\mathrm{MMT}$ & $\mathcal{L}_{\mathrm{SSC}} \& \mathcal{L}_{\mathrm{CE}}$ & $\boldsymbol{V}$ & $\mathrm{RQI}$ & Joint & 67.75 & 60.79 & 56.59 & 53.63 & 66.23 \\
10 & $\mathrm{MMT}$ & $\mathcal{L}_{\mathrm{SSC}} \rightarrow \mathcal{L}_{\mathrm{CE}}[26]$ & $\boldsymbol{X}$ & $\mathrm{RQI}$ & Pretrain-Finetune & 65.33 & 57.39 & 52.63 & 49.20 & 64.21 \\
\hline 11 & $\mathrm{MMT}$ & $\mathcal{L}_{\mathrm{DMT}}[60] \& \mathcal{L}_{\mathrm{CE}}$ & $\boldsymbol{X}$ & $\mathrm{RQI}$ & Alternate & 68.11 & 60.70 & 56.23 & 53.10 & 66.59 \\
\hline
\end{tabular}

Table B: Ablations Study of ConClaT. Scaling denotes whether scaling factor $\alpha$ was used. N-Type defines the type of negatives used from Image (I), Question (Q) and Random (R). All experiments are run with Back Translation data. 


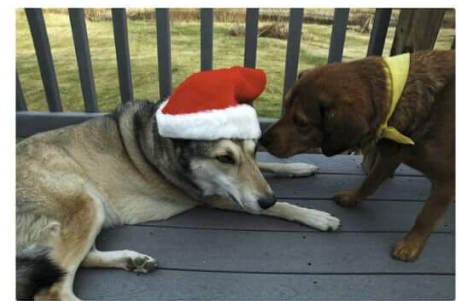

Q Who is sniffing who?

Q1 Somebody is being sniffed by who?

Q2 Who is doing the sniffing?

Q3 What is doing the sniffing?

GT dog

Avg. CS $0.16 / 1.00$

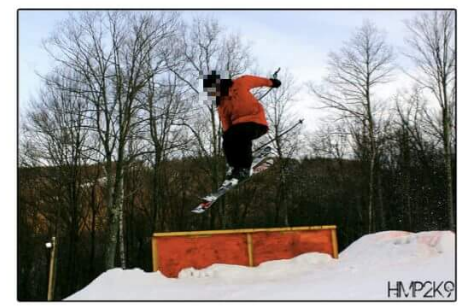

Q How many skis are on the ground?

Q1 What is the number of skis on the ground?

Q2 What is the ski count on the ground?

Q3 What's the exact number of skis on the ground?

GT

Avg. CS $0.16 / 1.00$

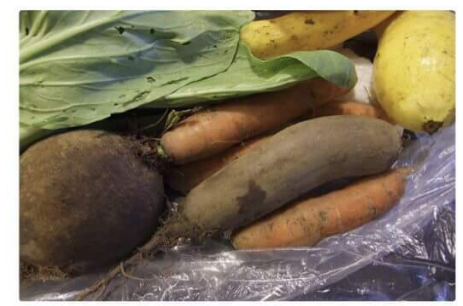

Q Are these fruit or vegetable?

food

Q1 Are these classified as fruit or vegetable?

Q2 Would these be called fruit or vegetable?

Q3 Do these count as fruit or

vegetable?

GT vegetable

Avg. CS $0.00 / 1.00$

carrots

vegetable

vegetables

vegetable

food

vegetable

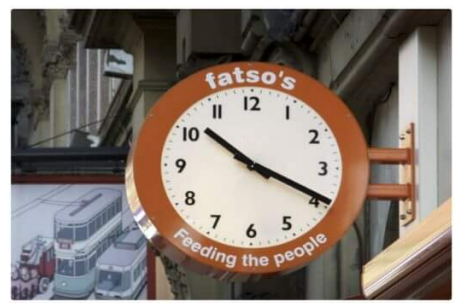

What color is the clock?

Can you tell me what color the clock is?

What color is the pictured clock?

orange

orange

yes

orange

orange

orange

What is the color of the clock? orange

orange

$0.37 / 1.00$

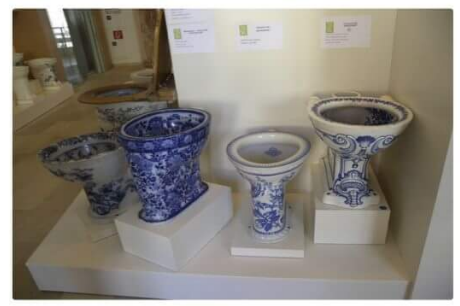

Which corner of the table is in

the frame?

What corner of the table is

shown?

Which corner of this table is

visible?

Which corner of the table is in

the shot?

left

$0.00 / 1.00$

top
left
wall
left
top right
left
top
left

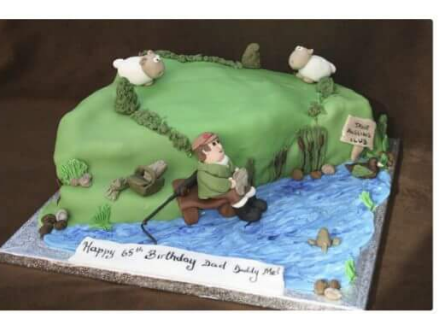

Is this food sweet?

Does this food have a sweet

taste?

Does this food teste sweet?

Is this a food that tastes sweet?

yes

yes $0.16 / 1.00$

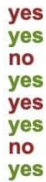

yes

no

yes

no

yes

$16 / 1.00$

Ours

Baseline

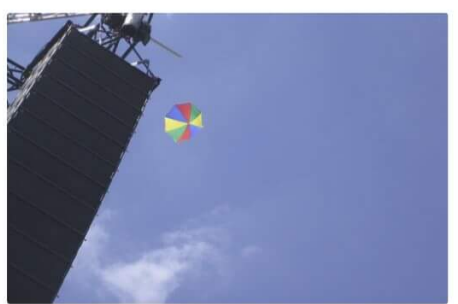

What color is the umbrella? blue

The color of the umbrella is rainbow rainbow The umbrella's color is what? blue The umbrella is what color? blue rainbow $0.16 / 1.00$

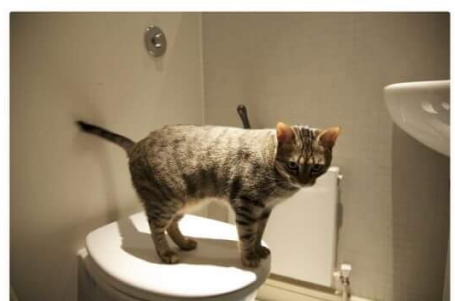

Is the cat able to access the toilet water?

as yes

Does the cat have access to the yes

toilet water?

s accessing the toilet water no

something the cat can do?

$0.16 / 1.00$

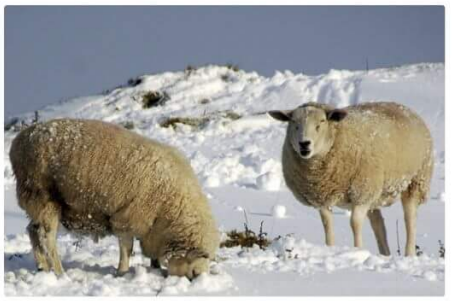

How many sheep are there?

2

What is the total of sheep?

sheep

What is the number of sheep?

How many sheep?

$0.37 / 1.00$

Figure B: Qualitative Examples. Predictions of ConClaT and MMT+CE baseline on several image-question pairs and their corresponding rephrased questions. Average Consensus Scores $(\mathrm{k}=1-4)$ are also shown at the bottom (higher the better). 


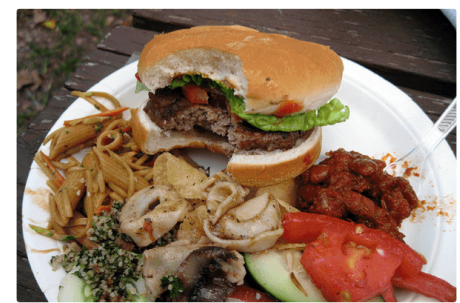

Q Is there cheese on the sandwich?

Q1 Does the sandwich have cheese?

Q2 The sandwich, does it have cheese?

Q3 Is there cheese on it?

GT no

Avg. CS $0.00 / 1.00$

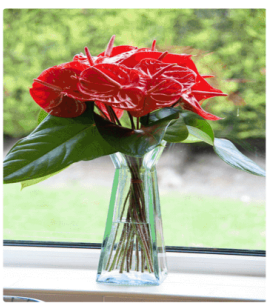

Q Do these flowers have yellow leaves?

Q1 Are there yellow leaves on these flowers?

Q2 Can yellow covered leaves be found on these flowers?

Q3 Are any of the leaves on these flowers yellow in color?

GT no

Avg. CS $0.37 / 1.00$

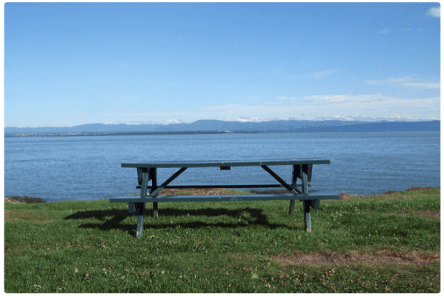

Q What color is the table?

green

Q1 Out of all the colors of the rainbow, which applies to the table?

The table is what color? is? is?

g. CS $0.37 / 1$.

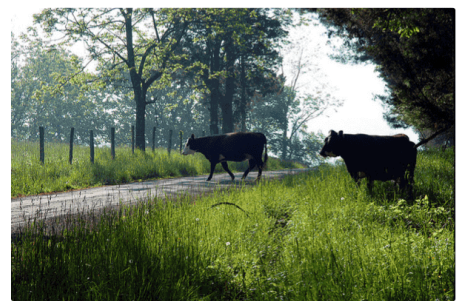

How many cows?

What is the count of cows in this picture?

How many cows are there in this picture?

How many cows can be seen?

2

$0.37 / 1.00$

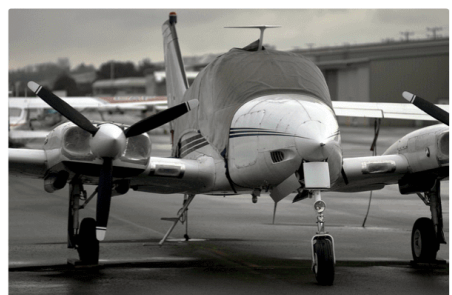

Is this planes color black?

Is the color of this plane black?

Is the plane's color black?

yes

yes

yes

no

Is the color associated with the plane black?

no $0.00 / 1.00$

no

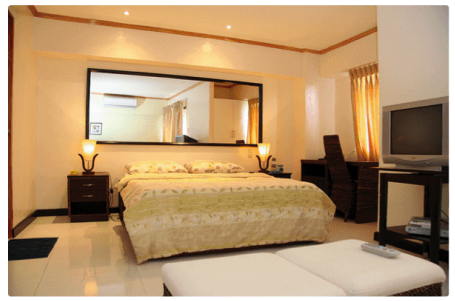

Do the pillows match the

bedspread?
Are the pillows matching th bedspread?

bedspread?

pillows?

pillows?

is the pattern on pillo

yes
$0.37 / 1.00$

$37 / 1.00$

yes

yes

no

yes

yes

yes
yes

Ours

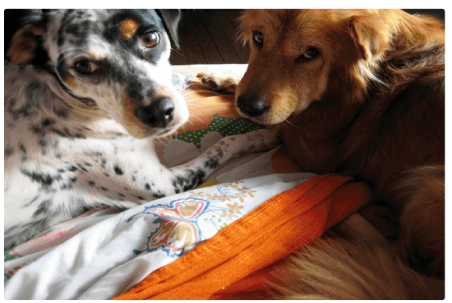

How many dogs are there?

2

What is the count for the amoun of dogs?

What is the number of dogs?

What is the amount of dogs?

$\mathbf{0 . 3 7 / 1 . 0 0}$

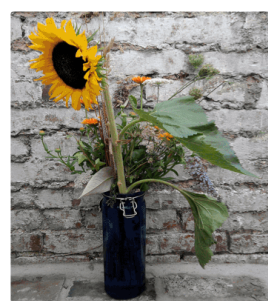

What is in the vase?

flowers

The vase has what in it?

What item is placed inside the vase?

The vase has what item in it?

glass

flowers

flowers

flowers

vase

flowers

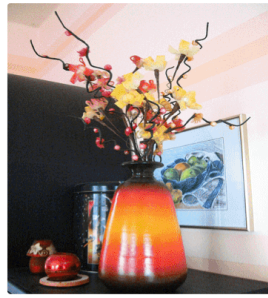

Is this a vase? yes

Does the picture depict a vase? no

yes

Is this a flower vase? yes

Does the photo show a vase? yes

yes
$0.37 / 1.00$

Figure C: Qualitative Examples. Predictions of ConClaT and MMT+CE baseline on several image-question pairs and their corresponding rephrased questions. Average Consensus Scores $(\mathrm{k}=1-4)$ are also shown at the bottom (higher the better). 

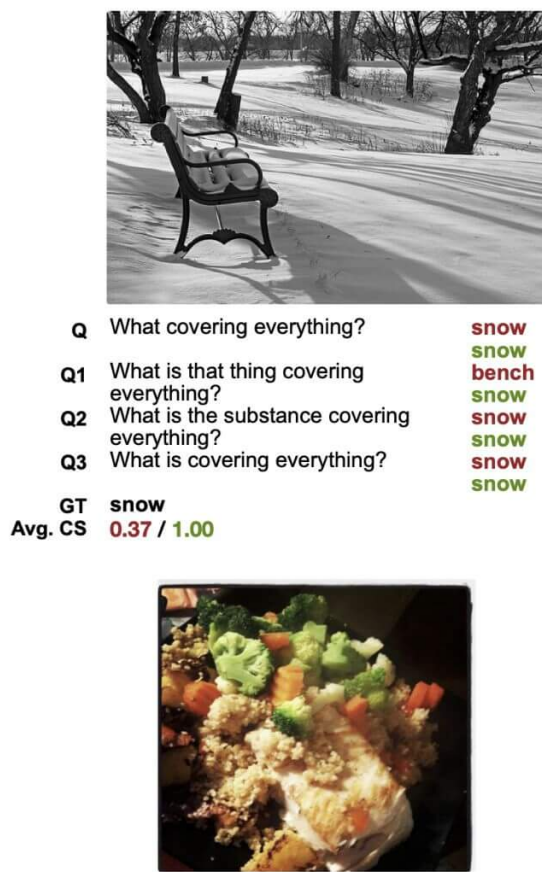

Q What is on the plate?

Q1 The plate has what on it?

Q2 Can you tell me what is on top of the plate?

Q3 What does the plate contain?

GT food

Avg. CS $0.16 / 0.37$

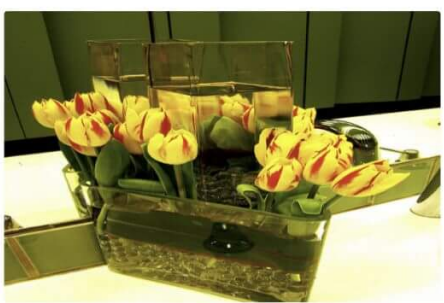

Q Are the flowers all one color?

Q1 Do the flowers come in one color?

Q2 Are the flowers one color?

Q3 Are the flowers in a single color?

GT no

Avg. CS $0.37 / 0.37$

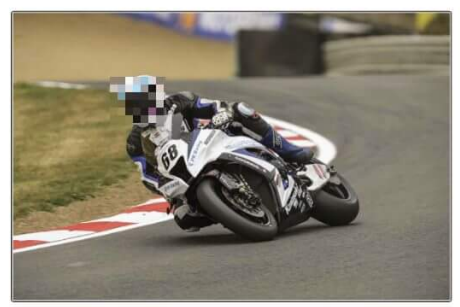

What colors are the stripes red and white on the left? red and white Can you name the colors yes on the far left?

The left side has which

colors?

red and white

red and white red and white What colors do you see on red and white the left? red and white $0.37 / 1.00$

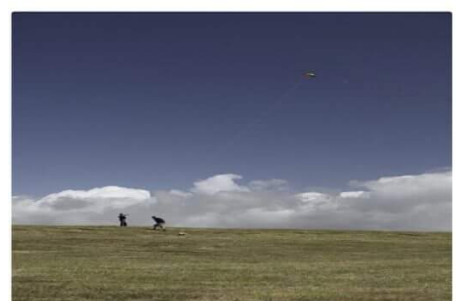

How many people are there?

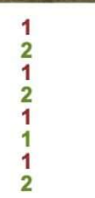

What number of people are there?

What is the count of people?

What is the amount of people?

2

$0.00 / 0.37$

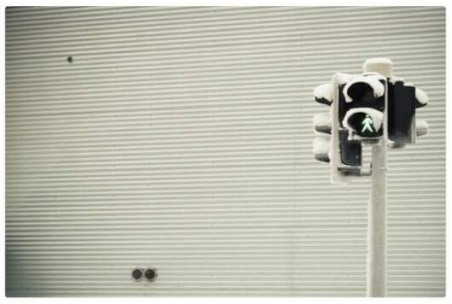

What does the sign say?

go

What is on the sign?

What does the sign indicate?

traffic lights

walk

What is written on the sign?

go

go

walk

$0.00 / 0.16$

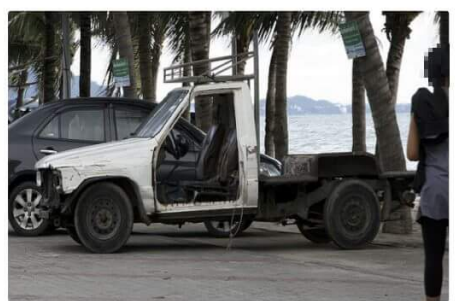

What kind of road is the truck paved parked on? asphalt For the truck, what kind of road concrete is it parked on? asphalt Can you identify the type of road no
the truck is parked on? Name the kind of road that truck paved is parked on? parking lot

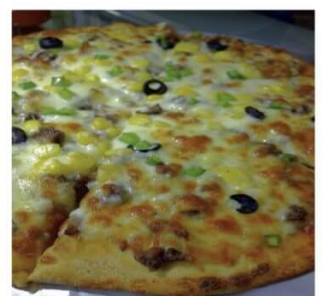

How many slices is this pizza cut 4 into?

The pizza has been cut into how many slices?

What amount of slices make up the cut pizza?

The amount of slices the pizza has been cut into is what?

6

$0.00 / 0.16$

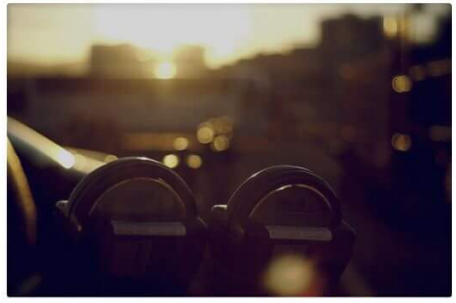

How many vehicles could use this object?

How many vehicles use this object?

Can you tell me how many

vehicles use this object? Will you tell me how many
vehicles can use this object?

$0.16 / 0.37$

Ours Baseline

Figure D: Qualitative Examples. Predictions of ConClaT and MMT+CE baseline on several image-question pairs and their corresponding rephrased questions. Average Consensus Scores $(\mathrm{k}=1-4)$ are also shown at the bottom (higher the better). 


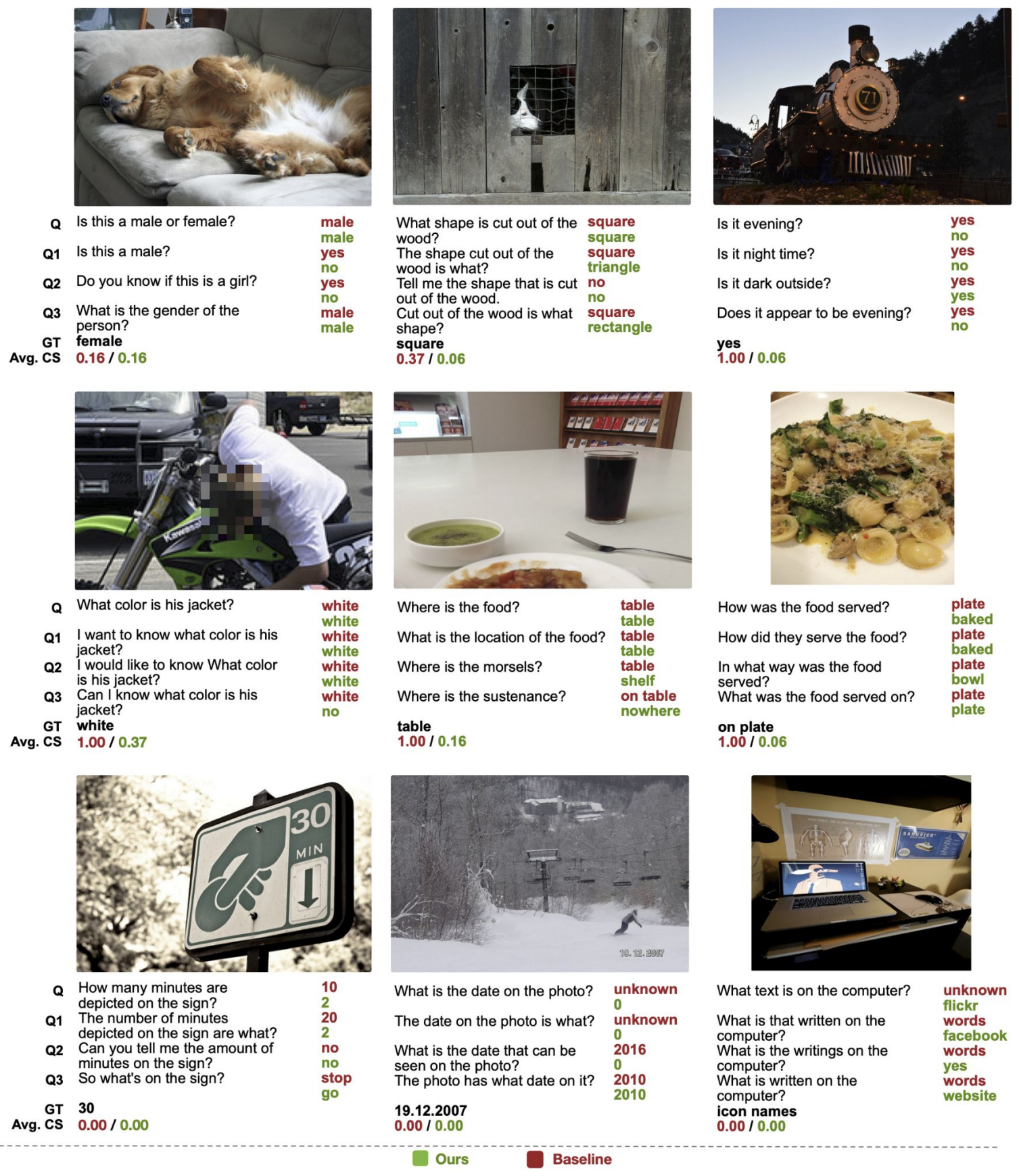

Figure E: Qualitative Examples. Predictions of ConClaT and MMT+CE baseline on several image-question pairs and their corresponding rephrased questions. Average Consensus Scores $(\mathrm{k}=1-4)$ are also shown at the bottom (higher the better). 


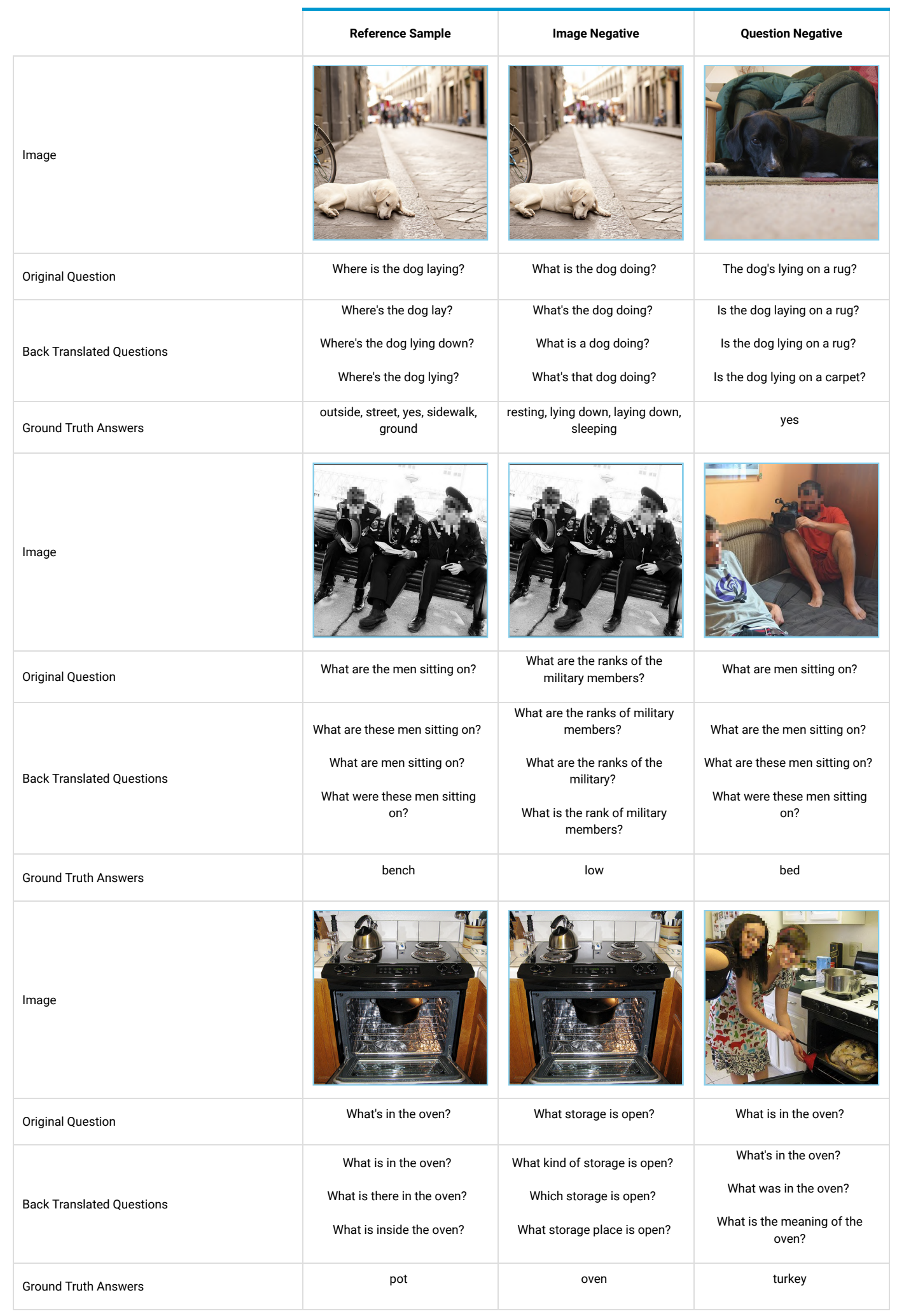

Figure F: Visualizing the triplets of samples from VQA dataset with corresponding mined Image and Question Negatives. 


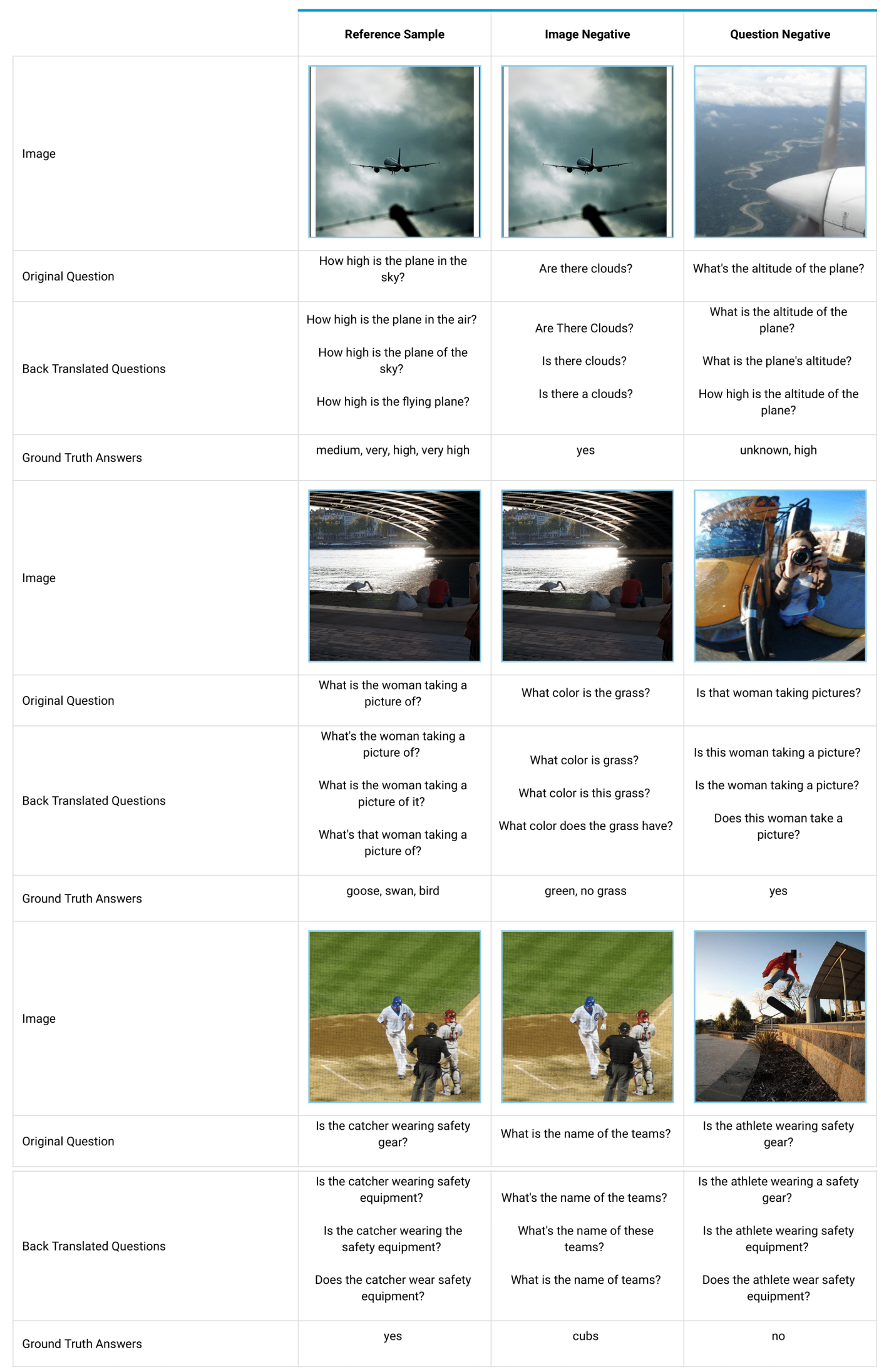

Figure G: Visualizing the triplets of samples from VQA dataset with corresponding mined Image and Question Negatives. 


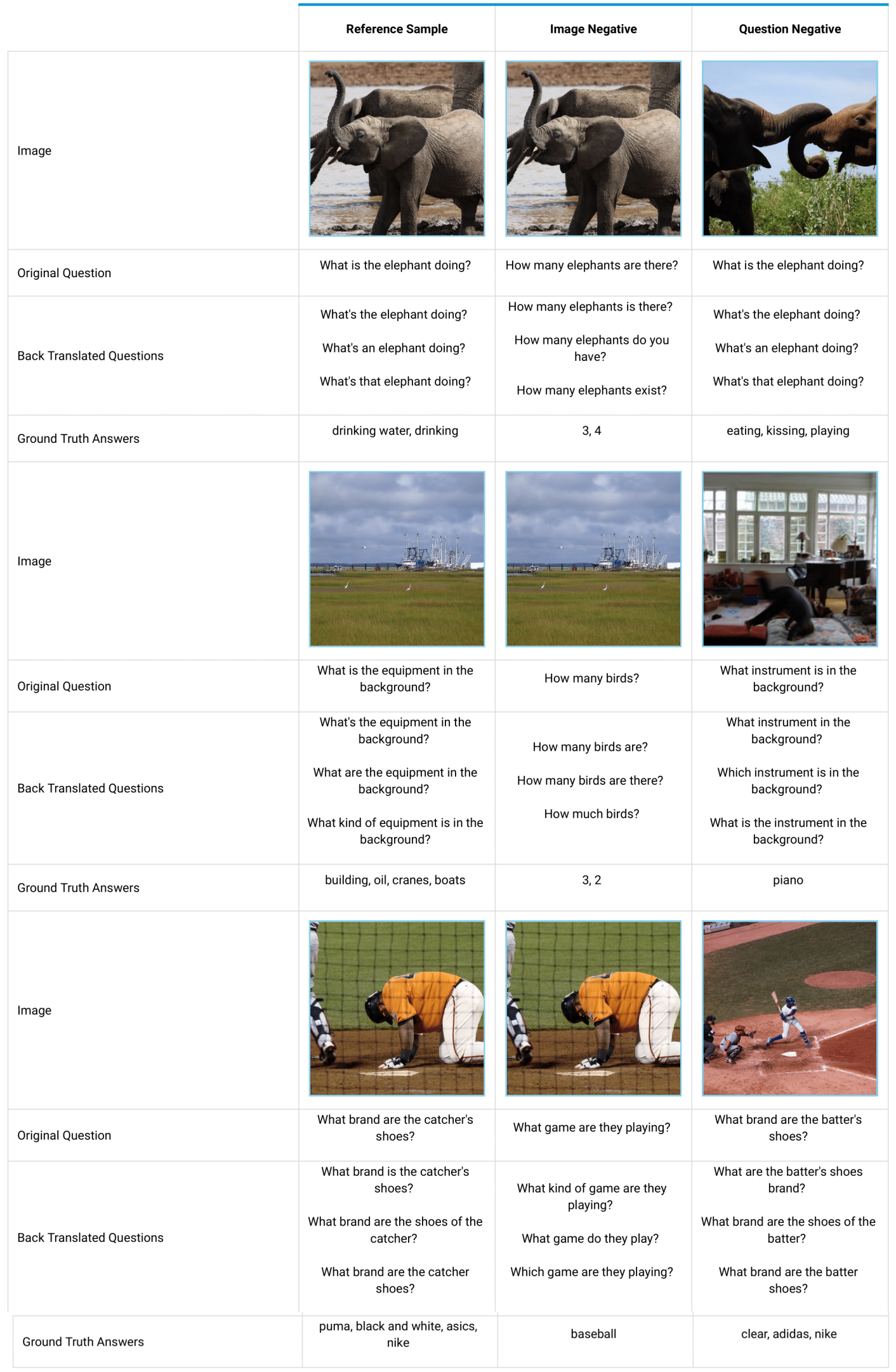

Figure H: Visualizing the triplets of samples from VQA dataset with corresponding mined Image and Question Negatives. 